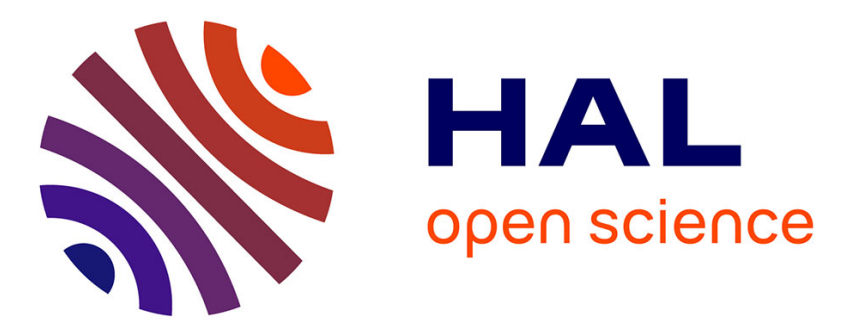

\title{
A qualitative study of linear drift-diffusion equations with time-dependent or degenerate coefficients
}

Jean-Philippe Bartier, Jean Dolbeault, Reinhard Illner, Michal Kowalczyk

\section{To cite this version:}

Jean-Philippe Bartier, Jean Dolbeault, Reinhard Illner, Michal Kowalczyk. A qualitative study of linear drift-diffusion equations with time-dependent or degenerate coefficients. Mathematical Models and Methods in Applied Sciences, 2007, 17 (3), pp.327-362. hal-00016363

\section{HAL Id: hal-00016363 https://hal.science/hal-00016363}

Submitted on 29 Dec 2005

HAL is a multi-disciplinary open access archive for the deposit and dissemination of scientific research documents, whether they are published or not. The documents may come from teaching and research institutions in France or abroad, or from public or private research centers.
L'archive ouverte pluridisciplinaire HAL, est destinée au dépôt et à la diffusion de documents scientifiques de niveau recherche, publiés ou non, émanant des établissements d'enseignement et de recherche français ou étrangers, des laboratoires publics ou privés. 


\title{
A QUALITATIVE STUDY OF LINEAR DRIFT-DIFFUSION EQUATIONS WITH TIME-DEPENDENT OR DEGENERATE COEFFICIENTS
}

\author{
Jean-Philippe Bartier, Jean Dolbeault \\ Ceremade, Université Paris Dauphine, Place de Lattre de Tassigny, \\ F-75775 Paris Cédex 16, France \\ bartier,dolbeaul@ceremade.dauphine.fr \\ Reinhard Illner \\ Mathematics and Statistics, University of Victoria, Clearihue Building D240, \\ 3800 Finnerty Road (Ring Road), Victoria, B.C., Canada \\ rillner@math.uvic.ca \\ Michał Kowalczyk \\ Universidad de Chile, Facultad de Ciencias Fisicas y Matemáticas, Depto Ingeniería \\ Matemática, Blanco Encalada 2120, Piso 5, Santiago, Chile \\ kowalczy@dim.uchile.cl
}

This paper is concerned with entropy methods for linear drift-diffusion equations with explicitly time-dependent or degenerate coefficients. Our goal is to establish a list of various qualitative properties of the solutions. The motivation for this study comes from a model for molecular motors, the so-called Brownian ratchet, and from a nonlinear equation arising in traffic flow models, for which complex long time dynamics occurs. General results are out of the scope of this paper, but we deal with several examples corresponding to most of the expected behaviors of the solutions.

We first prove a contraction property for general entropies which is a useful tool for uniqueness and for the convergence to some eventually time-dependent large time asymptotic solutions. Then we focus on power law and logarithmic relative entropies. When the diffusion term is of the type $\nabla\left(|x|^{\alpha} \nabla \cdot\right)$, we prove that the inequality relating the entropy with the entropy production term is a Hardy-Poincaré type inequality, that we establish. Here we assume that $\alpha \in(0,2]$ and the limit case $\alpha=2$ appears as a threshold for the method. As a consequence, we obtain an exponential decay of the relative entropies. In the case of time-periodic coefficients, we prove the existence of a unique time-periodic solution which attracts all other solutions.

The case of a degenerate diffusion coefficient taking the form $|x|^{\alpha}$ with $\alpha>2$ is also studied. The Gibbs state exhibits a non integrable singularity. In this case concentration phenomena may occur, but we conjecture that an additional time-dependence restores the smoothness of the asymptotic solution.

Keywords: Entropy method; relative entropy; convex entropy; contraction; large time asymptotics; stationary solutions; periodic solutions; drift-diffusion equation; logarithmic Sobolev inequality; convex Sobolev inequalities; Poincaré inequality; Caffarelli-KohnNirenberg inequalities; Hardy-Poincaré inequality; Csiszár-Kulback inequality; uniqueness; large time asymptotic behavior; convergence; time-periodic solutions; singular solutions; time-dependent drift; time-dependent diffusion coefficient; entropy - entropy production method.

AMS Subject Classification: 35K10, 47J20, 46E35; 35K15, 26D10, 35K20, 35K65 


\section{Introduction}

In this paper we consider explicitly time-dependent linear drift-diffusion equations which are motivated by traffic flow or Brownian ratchet models. We also study the case of degenerate diffusion coefficients, which are for instance of interest for traffic flow questions. To start with let us first describe these models in more details.

Generally speaking Brownian ratchets are models of phenomena in which directed motion in a periodic medium occurs in the presence of unbiased (on average) forces and interactions. In recent years several examples that vary from molecular motors and molecular pumps to Janossy effect in liquid crystals have been studied $1,6,21,31,32$. A simple model of a Brownian ratchet is the so-called flashing ratchet 20,28,29,16 given by the following Fokker-Planck type equation:

$$
\begin{array}{rlrl}
\sigma u_{x x}+\left(\phi_{x} u\right)_{x}=u_{t}, & & 0<x<1, \quad t>0, \\
u_{x}+\phi_{x} u=0, & x=0, \quad x=1, \\
u(x, 0)=u_{0}, & \int_{0}^{1} u_{0}=1,
\end{array}
$$

where $\phi=\phi(x, t)$ is periodic in both $x$ and $t$. As it turns out ${ }^{29}$ the long time behavior of the flashing ratchet is described by the periodic (in $t$ and of the same period as $\phi)$ solution $u_{\infty}(x, t)$. It was shown further in ${ }^{29}$ that $u_{\infty}$ is unique and globally attracting. These facts are basic in showing that transport is induced in the flashing ratchet model. In this paper we use the entropy methods to show the existence, uniqueness and stability of $u_{\infty}$ and generalize some preliminary results given in ${ }^{20}$. This approach is a significant simplification of that of ${ }^{29}$.

The homogeneous Fokker-Planck model of traffic dynamics as presented in 26 corresponds to

$$
u_{t}=\left(\sigma(t, x) u^{\prime}-\beta(t, x) u\right)^{\prime}, \quad(t, x) \in \mathbb{R}^{+} \times(0,1) .
$$

The variable $x$ represents the velocity of cars on a highway and $u$ is the corresponding distribution function. Spatial effects are not taken into account. The coefficients $\sigma$ and $\beta$ are defined in a nonlinear way as functions of the average velocity

$$
\bar{x}(t)=\frac{1}{\rho} \int_{0}^{1} x u(t, x) d x, \quad \text { with } \quad \rho=\int_{0}^{1} u(t, x) d x,
$$

by

$$
\beta(t, x)= \begin{cases}-C_{B}|x-\bar{x}(t)|^{2} \rho\left(1-\left|\frac{x-\bar{x}(t)}{1-\bar{x}(t)}\right|^{\delta}\right) & \text { if } \quad x>\bar{x}(t) \\ C_{A}|x-\bar{x}(t)|^{2}(1-\rho) & \text { if } \quad x \leq \bar{x}(t)\end{cases}
$$

and

$$
\sigma(t, x)=\sigma_{0} m_{1}(\rho) m_{2}(\bar{x}(t))|x-\bar{x}(t)|^{\gamma}
$$


for some positive constants $\sigma_{0}, C_{A}, C_{B}$ ( $A$ and $B$ respectively stand for Acceleration and Braking), and nonnegative exponents $\gamma$ and $\delta$. In ${ }^{26} m_{1}$ and $m_{2}$ are two continuous functions and the exponent $\gamma$ is assumed to be smaller than 3 . The parameter $\rho$ is the total density of the traffic and does not depend on $t$. We refer to ${ }^{25,19}$ for further comments on this model.

The nonlinear dependence of $\sigma$ and $\beta$ on the solution $u$ makes the analysis difficult. Moreover relevant phenomena for traffic modeling apparently occur on time scales much smaller than the ones of the asymptotic regimes. The degeneracy of the diffusion coefficient corresponds to an idealized situation since one can always argue for the presence of some "residual" diffusion. It seems however that the degeneracy of $\sigma$ makes sense at least to derive a picture at first order. The heuristic idea is that drivers adapt their speed to the mean speed of the traffic and the diffusion accounts for stochastic errors they make in their evaluation of $\bar{x}$. The closer they are to $\bar{x}$ and the smaller are the errors.

In this paper, as a rough approximation, we dont take nonlinearities into account but focus on the qualitative properties of the solutions due to the degeneracy of the diffusion coefficient and the time dependence of $\sigma$ and $\beta$. The time dependence in traffic flow models is definitely relevant. As a special case, the time-periodic regime is of particular interest because of its connections with the phenomenon of "stop-and-go" waves ${ }^{26,23}$.

For time-dependent as well as for degenerate coefficients, our approach is based on entropy estimates. The so-called entropy - entropy production method has received a lot of attention both in the linear and the nonlinear framework $35,5,12,3$ during the last few years. Alternative approaches based on variational methods ${ }^{18}$, mass transportation techniques ${ }^{27,33,37}$ and hypercontractivity ${ }^{22,9}$ have also been developed. Since we deal with linear equations, there is some freedom in the choice of the entropy and whenever possible we use general convex entropies ${ }^{5,4}$.

This paper is organized as follows. We begin with general drift-diffusion equations and state the underlying contraction property which gives uniqueness and convergence to an asymptotic state. Although stated at a somewhat formal level, these results emphasize a fundamental property which is common to all special cases studied in this paper. Section 2 is devoted to equations with degenerate diffusion coefficients. We work on a caricature of a traffic flow equation and prove a HardyPoincaré inequality which relates entropy and entropy production, and therefore governs the long time behaviour. Section 3 is devoted to equations with explicitly time-dependent periodic coefficients. We prove the existence of a unique periodic asymptotic solution which attracts all solutions to the Cauchy problem. The examples of this section mostly refer to molecular motors. In the last section, we give several examples which mix the features observed in the two previous sections and provide a qualitative understanding of some phenomena that are numerically observed in traffic flow models.

In the applications to traffic flow and Brownian ratchets models, the dimension is 
one in most cases. However, our results hold for any dimension and should apply to other types of models, see, e.g., ${ }^{15}$. The two most striking points of our approach are: 1) general time-periodic forces can be considered, 2) the diffusion coefficient may be degenerate. This gives rise to power law weights in the Hardy-Poincaré inequality relating the entropy and the entropy production. We prove this inequality from a variational point of view. The proof of such a result by any of the other techniques mentioned above is as far as we know still open, except for the entropy - entropy production method, in some special cases, as we shall see in the last section.

\section{General drift-diffusion equations}

Consider the drift-diffusion equation

$$
u_{t}=\nabla \cdot[\sigma(x, t) \nabla u+F(x, t) u]
$$

for $t \geq 0, x \in \Omega$, where $\Omega$ is a domain in $\mathbb{R}^{d}, d \geq 1$. Here $\Omega$ is not necessarily bounded. Assume further that $u$ satisfies Robin, or no flux, boundary conditions:

$$
[\sigma(x, t) \nabla u+F(x, t) u] \cdot \nu(x)=0 \quad \forall(x, t) \in \partial \Omega \times \mathbb{R}^{+}
$$

if $\Omega \not \equiv \mathbb{R}^{d}$. Here $\nu(x)$ is the outgoing normal unit vector at $x \in \partial \Omega$. For any $(x, t) \in \Omega \times \mathbb{R}^{+}$, the diffusion matrix $\sigma(x, t)$ is assumed to be a nonnegative definite symmetric matrix. In most cases we will take it proportional to the identity, up to a scalar coefficient that will be called the diffusion coefficient. The drift force $F(x, t)$ does not depend on $u$ and is given, so that (1.1) is a linear equation. Our main tool is the relative entropy of $u_{1}$ with respect to $u_{2}$, defined by

$$
\mathrm{e}_{\psi}\left[u_{1} \mid u_{2}\right]:=\int_{\Omega} \psi\left(\frac{u_{1}}{u_{2}}\right) u_{2} d x
$$

for an appropriately chosen convex function $\psi$. Although some of our tools are adequate for sign changing solutions (take for instance $\psi(v)=|v-1|^{2}$ in that case), for simplicity we will restrict our study to nonnegative solutions of (1.1). Some results of this section are written only at a formal level in the sense that we assume that the solutions have sufficient regularity and decay properties to justify all computations as if they were smooth, and compactly supported in case of unbounded domains. The point is to give generic results which can be justified with all details in each of the examples of this paper.

\subsection{A contraction property}

Theorem 1.1. Let $\psi$ be a strictly convex function on $\mathbb{R}^{+}$, bounded from below and such that $\psi(1)=\psi^{\prime}(1)=0$. Consider two smooth global in $t$ nonnegative solutions $u_{1}$ and $u_{2}$ of (1.1)-(1.2) with initial data $u_{1}^{0}$ and $u_{2}^{0}$ in $L_{+}^{1}(\Omega)$ such that $\psi\left(u_{1}^{0} / u_{2}^{0}\right) u_{2}^{0}$ also belongs to $L^{1}(\Omega)$. Assume that $u_{2}(\cdot, t)$ is positive for any $t \in \mathbb{R}^{+}$and $\int_{\Omega} u_{1}^{0} d x=$ 
$\int_{\Omega} u_{2}^{0} d x$. If $\Omega$ is unbounded and if $u_{1}$ and $u_{2}$ decay fast enough at infinity, or if $\Omega$ is bounded, then, for any $t>0$,

$$
\begin{gathered}
0 \leq e_{\psi}\left[u_{1}(\cdot, t) \mid u_{2}(\cdot, t)\right] \leq e_{\psi}\left[u_{1}^{0} \mid u_{2}^{0}\right] \\
\frac{d}{d t} e_{\psi}\left[u_{1}(\cdot, t) \mid u_{2}(\cdot, t)\right]=-\int_{\Omega} \psi^{\prime \prime}\left(\frac{u_{1}}{u_{2}}\right) u_{2}\left(\nabla\left(\frac{u_{1}}{u_{2}}\right) \cdot \sigma \nabla\left(\frac{u_{1}}{u_{2}}\right)\right) d x .
\end{gathered}
$$

By sufficient decay at infinity and by smooth solutions, we simply mean that all integrations by parts needed in the proof make sense.

Proof. The lower estimate on $\mathrm{e}_{\psi}\left[u_{1}(\cdot, t) \mid u_{2}(\cdot, t)\right]$ is a consequence of Jensen's inequality: for any two functions $U_{1}, U_{2} \in L_{+}^{1}(\Omega)$ such that $\int_{\Omega} U_{1} d x=\int_{\Omega} U_{2} d x$, we have

$$
\frac{e_{\psi}\left[U_{1} \mid U_{2}\right]}{\int_{\Omega} U_{2} d x}=\int_{\Omega} \psi\left(\frac{U_{1}}{U_{2}}\right) \frac{U_{2} d x}{\int_{\Omega} U_{2} d x} \geq \psi\left(\int_{\Omega} \frac{U_{1}}{U_{2}} \frac{U_{2} d x}{\int_{\Omega} U_{2} d x}\right)=\psi(1)=0,
$$

with equality if and only if $U_{1}=U_{2}$ a.e. The inequality holds as a special case if $U_{1}=u_{1}(\cdot, t)$ and $U_{2}=u_{2}(\cdot, t)$, since $\int_{\Omega} u_{1}(\cdot, t) d x=\int_{\Omega} u_{2}(\cdot, t) d x$ is independent of $t \geq 0$.

The upper bound relies on the entropy decay which follows from the following computation:

$$
\begin{aligned}
& \frac{d}{d t} \mathrm{e}_{\psi}\left[u_{1}(\cdot, t) \mid u_{2}(\cdot, t)\right] \\
& =\int_{\Omega}\left[\psi^{\prime}\left(\frac{u_{1}}{u_{2}}\right)\left(u_{1}\right)_{t}+\left(\psi\left(\frac{u_{1}}{u_{2}}\right)-\psi^{\prime}\left(\frac{u_{1}}{u_{2}}\right) \frac{u_{1}}{u_{2}}\right)\left(u_{2}\right)_{t}\right] d x \\
& =-\int_{\Omega}\left[\psi^{\prime \prime}\left(\frac{u_{1}}{u_{2}}\right) \nabla\left(\frac{u_{1}}{u_{2}}\right) \cdot\left(\sigma \nabla u_{1}+F u_{1}\right)\right. \\
& \left.\quad-\psi^{\prime \prime}\left(\frac{u_{1}}{u_{2}}\right) \frac{u_{1}}{u_{2}} \nabla\left(\frac{u_{1}}{u_{2}}\right) \cdot\left(\sigma \nabla u_{2}+F u_{2}\right)\right] d x \\
& =-\int_{\Omega}\left[\psi^{\prime \prime}\left(\frac{u_{1}}{u_{2}}\right) \nabla\left(\frac{u_{1}}{u_{2}}\right) \cdot \sigma\left(\nabla u_{1}-\frac{u_{1}}{u_{2}} \nabla u_{2}\right)\right] d x .
\end{aligned}
$$

The results of Theorem 1.1 can be extended to larger classes of solutions using the integral form of (1.3):

$$
\begin{array}{r}
\mathrm{e}_{\psi}\left[u_{1}(\cdot, t) \mid u_{2}(\cdot, t)\right]+\int_{0}^{t} \int_{\Omega} \psi^{\prime \prime}\left(\frac{u_{1}}{u_{2}}\right) u_{2}\left(\nabla\left(\frac{u_{1}}{u_{2}}\right) \cdot \sigma \nabla\left(\frac{u_{1}}{u_{2}}\right)\right) d x d s \\
=\mathrm{e}_{\psi}\left[u_{1}^{0} \mid u_{2}^{0}\right] \quad \forall t \geq 0,
\end{array}
$$

and using convexity and lower semi-continuity properties. By regularizing the initial data and the coefficients of the equation, we can prove such an estimate for approximating solutions. Passing to the limit, if no further a priori estimates are 
available and if the second term of the left hand side is lower semi-continuous, the above identity generically becomes an inequality:

$$
\begin{array}{r}
\mathrm{e}_{\psi}\left[u_{1}(\cdot, t) \mid u_{2}(\cdot, t)\right]+\int_{0}^{t} \int_{\Omega} \psi^{\prime \prime}\left(\frac{u_{1}}{u_{2}}\right) u_{2}\left(\nabla\left(\frac{u_{1}}{u_{2}}\right) \cdot \sigma \nabla\left(\frac{u_{1}}{u_{2}}\right)\right) d x d s \\
\leq \mathrm{e}_{\psi}\left[u_{1}^{0} \mid u_{2}^{0}\right] \quad \forall t \geq 0
\end{array}
$$

for any weak solution of (1.1)-(1.2) which is obtained by an approximating procedure. It is out of the scope of this paper to give optimal conditions for obtaining (1.6), but in all special cases where this procedure can be applied, we will simply refer to (1.6) without further justification.

\subsection{Uniqueness}

The contraction property has rather simple but interesting consequences concerning the uniqueness of the solutions to the Cauchy problem and stationary solutions. Let $\mathbb{I}_{d}$ be the identity matrix in $\mathbb{R}^{d} \times \mathbb{R}^{d}$. Assume that the following uniform ellipticity condition is verified: for any $(x, t) \in \Omega \times \mathbb{R}^{+}$,

There exists a positive function $\sigma_{0}$ such that $\sigma(x, t) \geq \sigma_{0}(x) \mathbb{I}_{d}$.

Corollary 1.1. Under the assumptions of Theorem 1.1 and (1.7), if (1.6) holds for any solution $u$, then (1.1)-(1.2) supplemented with an initial data $u^{0}$ has at most one smooth positive global solution.

Proof. If there were two solutions $u_{1}$ and $u_{2}$ corresponding to the same initial data $u_{1}^{0}=u_{2}^{0}=u^{0}$, then by Jensen's inequality $(1.4), 0 \leq \mathrm{e}_{\psi}\left[u_{1}(\cdot, t) \mid u_{2}(\cdot, t)\right]$ for any smooth strictly convex function $\psi$. On the other hand,

$$
\mathrm{e}_{\psi}\left[u_{1}(\cdot, t) \mid u_{2}(\cdot, t)\right] \leq \mathrm{e}_{\psi}\left[u_{1}^{0} \mid u_{2}^{0}\right]=0
$$

for any $t \geq 0$, by (1.6). The equality case in Jensen's inequality means: $u_{1} \equiv u_{2}$.

Corollary 1.2. Let $\Omega$ be a simply connected, bounded domain. Assume that $F$ is bounded, and $G(x, t):=(\sigma(x, t))^{-1} F(x, t)$ is independent of t and such that $G=\nabla \varphi$ for some bounded potential $\varphi$. Under Assumption (1.7), for any $M>0$, (1.1) has a unique positive bounded stationary solution $u_{\infty}$ such that $\int_{\Omega} u_{\infty} d x=M$, which moreover satisfies boundary conditions (1.2).

Proof. The function

$$
u_{2, \infty}:=M \frac{e^{-\varphi}}{\int_{\Omega} e^{-\varphi} d x}
$$

is a stationary solution. For any other stationary solution $u_{1, \infty}$, we may find a strictly convex function $\psi$, satisfying the conditions of Theorem 1.1, such that $\mathrm{e}_{\psi}\left[u_{1, \infty} \mid u_{2, \infty}\right]<\infty$ and (1.6) holds. By (1.6) we know that

$$
\int_{\Omega} \psi^{\prime \prime}\left(\frac{u_{1, \infty}}{u_{2, \infty}}\right) u_{2, \infty}\left(\nabla\left(\frac{u_{1, \infty}}{u_{2, \infty}}\right), \sigma \nabla\left(\frac{u_{1, \infty}}{u_{2, \infty}}\right)\right) d x=0 .
$$


On the unique connected component of $\Omega, u_{1, \infty}$ and $u_{2, \infty}$ are collinear: $u_{1, \infty}=$ $\lambda u_{2, \infty}$. The conditions $u_{1, \infty} \geq 0, u_{2, \infty} \geq 0, \int_{\Omega} u_{1, \infty} d x=\int_{\Omega} u_{2, \infty} d x$ imply $\lambda=1$.

If we don't assume that the stationary solution is bounded, then the question becomes more tricky. First of all, one has to find a convex function $\psi$ such that $\mathrm{e}_{\psi}\left[u_{1, \infty} \mid u_{2, \infty}\right]<\infty$. This can always be done. See ${ }^{10}$ for more details. Then one has to make sure that one can establish (1.6), which for stationary solutions is equivalent to (1.5). This is far from being obvious at our level of generality but is usually easy to implement in all practical cases of applications.

Boundedness from above of the potential $\varphi$ means that the unique stationary solution is positive which is convenient to define the relative entropy but not compulsory. Boundedness from below could be relaxed to the weaker assumption that $e^{-\varphi} \in L^{1}(\Omega)$.

The result of Corollary 1.2 can be extended to time-dependent asymptotic states, see ${ }^{20}$. In Section 3, by assuming that the dependence of the coefficients of Equation (1.1) is time-periodic, we will prove a result of existence of a time-periodic asymptotic solution, which is also unique because of the contraction property, and generalizes somes of the results contained in the above references.

\subsection{Large time behaviour: convergence to stationary solutions}

Assume that the following conditions are satisfied:

(i) For any $x \in \Omega, G(x, t):=(\sigma(x, t))^{-1} F(x, t)=-\nabla \varphi(x)$ is independent of $t$ and $\varphi$ is bounded.

(ii) The diffusion coefficient is not integrable in $t$, i.e.,

$$
\int_{0}^{+\infty} \sigma(x, t) d t=+\infty \quad \forall x \in \Omega \text { a.e. }
$$

If $\Omega$ is bounded, we can define $u_{\infty}(x):=M \frac{e^{-\varphi}}{\int_{\Omega} e^{-\varphi} d x}$.

Theorem 1.2. Under Assumptions (i)-(ii), if $\Omega$ is bounded and simply connected, the function $u_{\infty}$ is the unique bounded stationary solution of (1.1) with mass $M$. Moreover, let $u$ be any solution of (1.1) with a nonnegative initial data $u_{0}$ in $L^{1}(\Omega)$ such that $\left\|u_{0}\right\|_{L^{1}(\Omega)}=M$. Then there exists a convex function $\psi$ such that $e_{\psi}\left[u_{0} \mid u_{\infty}\right]$ is finite. Additionally, if (1.6) holds, then $u$ converges to $u_{\infty}$ in $L^{1}(\Omega)$ as $t \rightarrow+\infty$.

Proof. There exists a strictly convex function $\psi$ satisfying $\psi(1)=\psi^{\prime}(1)=0$ such that $\psi\left(u_{0} / u_{\infty}\right)$ is bounded in $L^{1}\left(\Omega, u_{\infty}(x) d x\right)$, i.e., $\mathrm{e}_{\psi}\left[u_{0} \mid u_{\infty}\right]<\infty$, see ${ }^{10}$. Note that the choice of $\psi$ may depend on $u_{0}$, if we do not make further assumptions on $u_{0}$. As a consequence of $(1.6), \nabla\left(u(\cdot, t) / u_{\infty}\right)$ converges to 0 as $t \rightarrow+\infty, x \in \Omega$ a.e. This proves simultaneously the uniqueness of the stationary solution, and the convergence of any other solution to $u_{\infty}$. 
This result is formal in the sense that for a given $u_{0}$, the existence of a function $\psi$ such that (1.6) holds is not easy to check. In practical situations, $\psi$ is given, such that the second term of the left hand side in (1.6) is lower semi-continuous as a function of $u$ and we require that $u_{0}$ is such that $\mathrm{e}_{\psi}\left[u_{0} \mid u_{\infty}\right]$ is finite. Then Inequality (1.6) holds for any reasonable solution $u$ obtained as a limit of an approximation procedure. The convergence of $u$ to $u_{\infty}$ in Theorem 1.2 has to be understood as

$$
\lim _{t \rightarrow \infty} \int_{t}^{t+1}\left\|u(\cdot, s)-u_{\infty}\right\|_{L^{1}(\Omega)} d s=0 .
$$

The existence of $\psi$ in the stationary case holds for the same reasons as in the proof of Corollary 1.2. There are several possible generalizations, for instance to the case of an unbounded domain $\Omega$, or to a time-dependent function $G$ which converges to some time-independent limit. The result of Theorem 1.2 can also be extended to asymptotic states which are time-dependent. In Section 3, when the dependence of the coefficients of Equation (1.1) is time-periodic, we will prove that all solutions to the Cauchy problem converge to a unique time-periodic asymptotic solution.

\subsection{Further elementary properties of convex entropies}

A more precise inequality than (1.4) can be established by doing a Taylor expansion to order two if $\psi^{\prime \prime}(v)$ compares with $v^{p-2}$. This is known as the Csiszár-Kullback inequality if $p=1^{17,30,36}$ and the generalized Csiszár-Kullback inequality (see ${ }^{18,10}$ ) if $p \in[1,2]$. For completeness, we give a precise statement with a proof, which is essentially taken from ${ }^{10}$.

Proposition 1.1. Assume that $\Omega$ is a domain in $\mathbb{R}^{d}$. Let $v_{1}$ and $v_{2}$ be two nontrivial nonnegative functions in $L^{1} \cap L^{p}(\Omega), p \in[1,2]$, and consider a nonnegative strictly convex function $\phi \in C^{2}(0,+\infty)$. If

$$
A:=\inf _{v>0} v^{2-p} \phi^{\prime \prime}(v)>0
$$

then

$$
\int_{\Omega}\left(\phi\left(v_{1}\right)-\phi\left(v_{2}\right)-\phi^{\prime}\left(v_{2}\right)\left(v_{1}-v_{2}\right)\right) d x \geq \frac{A}{2^{2 / p}} \frac{\left\|v_{1}-v_{2}\right\|_{L^{p}(\Omega)}^{2}}{\max _{i=1,2}\left\|v_{i}\right\|_{L^{p}(\Omega)}^{2-p}} .
$$

Proof. The case $p=1$ is the well known Csiszár-Kullback inequality, see for instance ${ }^{36}$. Assume first that $v_{1}>0$. By a Taylor expansion at order two, we get

$$
\begin{aligned}
\int_{\Omega}\left(\phi\left(v_{1}\right)-\phi\left(v_{2}\right)-\phi^{\prime}\left(v_{2}\right)\left(v_{1}-v_{2}\right)\right) d x= & \frac{1}{2} \int_{\Omega} \phi^{\prime \prime}(\xi)\left|v_{1}-v_{2}\right|^{2} d x \\
& \geq \frac{A}{2} \int_{\Omega} \xi^{p-2}\left|v_{1}-v_{2}\right|^{2} d x
\end{aligned}
$$

where $\xi$ lies between $v_{1}$ and $v_{2}$. By Hölder's inequality, for any $h>0$ and for any measurable set $\mathcal{A} \subset \Omega$, we get

$$
\int_{\mathcal{A}}\left|v_{1}-v_{2}\right|^{p} h^{-\alpha} h^{\alpha} d x \leq\left(\int_{\mathcal{A}}\left|v_{1}-v_{2}\right|^{2} h^{p-2} d x\right)^{p / 2}\left(\int_{\mathcal{A}} h^{\alpha s} d x\right)^{1 / s}
$$


with $\alpha=p(2-p) / 2, s=2 /(2-p)$. Thus

$$
\left(\int_{\mathcal{A}}\left|v_{1}-v_{2}\right|^{2} h^{p-2} d x\right)^{p / 2} \geq\left(\int_{\mathcal{A}}\left|v_{1}-v_{2}\right|^{p} d x\right)\left(\int_{\mathcal{A}} h^{p} d x\right)^{(p-2) / 2} .
$$

We apply this formula to two different sets.

i) On $\mathcal{A}=\mathcal{A}_{1}=\left\{x \in \Omega: v_{1}(x)>v_{2}(x)\right\}$, use $\xi^{p-2}>v_{1}^{p-2}$ and take $h=v_{1}$ :

$$
\left(\int_{\mathcal{A}_{1}}\left|v_{1}-v_{2}\right|^{2} \xi^{p-2} d x\right)^{p / 2} \geq\left(\int_{\mathcal{A}_{1}}\left|v_{1}-v_{2}\right|^{p} d x\right)\left\|v_{1}\right\|_{L^{p}(\Omega)}^{-(2-p) p / 2} .
$$

ii) On $\mathcal{A}=\mathcal{A}_{2}=\left\{x \in \Omega: v_{1}(x) \leq v_{2}(x)\right\}$, use $\xi^{p-2} \geq v_{2}^{p-2}$ and take $h=v_{2}$ :

$$
\left(\int_{\mathcal{A}_{2}}\left|v_{1}-v_{2}\right|^{2} \xi^{p-2} d x\right)^{p / 2} \geq\left(\int_{\mathcal{A}_{2}}\left|v_{1}-v_{2}\right|^{p} d x\right)\left\|v_{2}\right\|_{L^{p}(\Omega)}^{-(2-p) p / 2} .
$$

To prove the result in the case $v_{1}>0$, we just add the two previous inequalities and use the inequality $(a+b)^{r} \leq 2^{r-1}\left(a^{r}+b^{r}\right)$ for any $a, b \geq 0$ and $r \geq 1$. To handle the case $v_{1} \geq 0$, we proceed by density: apply the result to $v_{1}^{\epsilon}(x)=v_{1}(x)+\epsilon \exp \left(-|x|^{2}\right)$ and let $\epsilon \rightarrow 0$ using Lebesgue's convergence theorem.

The relative entropy $\mathrm{e}_{\psi}\left[u_{1} \mid u_{2}\right]$ is homogeneous of degree 1 which is not the case of $\int_{\Omega}\left(\phi\left(v_{1}\right)-\phi\left(v_{2}\right)-\phi^{\prime}\left(v_{2}\right)\left(v_{1}-v_{2}\right)\right) d x$. To get an estimate of $\left\|u_{1}-u_{2}\right\|_{L^{p}(\Omega)}$ in terms of $\mathrm{e}_{\psi}\left[u_{1} \mid u_{2}\right]$, we need a uniform upper bound on $u_{2}$.

Corollary 1.3. Assume that $\Omega$ is a domain in $\mathbb{R}^{d}$. Consider $u_{1} \in L_{+}^{1} \cap L^{p}(\Omega)$ with $p \in[1,2], u_{2} \in L_{+}^{1} \cap L^{\infty}(\Omega)$ and a nonnegative strictly convex function $\psi \in$ $C^{2}(0,+\infty)$ such that $\psi(1)=\psi^{\prime}(1)=0$. If

$$
A:=\inf _{v>0} v^{2-p} \psi^{\prime \prime}(v)>0 \text {, }
$$

then

$$
e_{\psi}\left[u_{1} \mid u_{2}\right] \geq \frac{A \min _{i=1,2}\left\|u_{i}\right\|_{L^{p}(\Omega)}^{p-2}}{2^{2 / p}\left\|u_{2}\right\|_{L^{\infty}(\Omega)}^{p-1}}\left\|u_{1}-u_{2}\right\|_{L^{p}(\Omega)}^{2} .
$$

Proof. First of all,

$$
\mathrm{e}_{\psi}\left[u_{1} \mid u_{2}\right]=\int_{\Omega} \psi\left(\frac{u_{1}}{u_{2}}\right) u_{2} d x \geq \int_{\Omega} \psi\left(\frac{u_{1}}{u_{2}}\right) \frac{u_{2}^{p}}{\left\|u_{2}\right\|_{L^{\infty}(\Omega)}^{p-1}} d x .
$$

Let $\phi(v):=A \frac{v^{p}}{p(p-1)}$. By quadrature, we get that

$$
\psi(v) \geq \phi(v)-\phi(1)-\phi^{\prime}(1)(v-1) \quad \forall v \in \mathbb{R}^{+},
$$

and may therefore apply Proposition 1.1 using the homogeneity of $\phi$ :

$$
\psi\left(\frac{u_{1}}{u_{2}}\right) u_{2}^{p} \geq \phi\left(u_{1}\right)-\phi\left(u_{2}\right)-\phi^{\prime}\left(u_{2}\right)\left(u_{1}-u_{2}\right) .
$$


A typical convex function $\psi$ for which (1.8) is satisfied is

$$
\psi_{p}(v):=\frac{v^{p}-1-p(v-1)}{p-1}
$$

for $p \in(1,2]$, and

$$
\psi_{1}(v):=v \log v-(v-1)
$$

in the limit case $p=1$. Although more general statements can be obtained for general convex functions $\psi$ satisfying (1.8), for simplicity we restrict now our estimates to the case $\psi=\psi_{p}$ for some $p \in[1,2]$. In $(1.9)$, we need some uniform estimate on $\left\|u_{1}\right\|_{L^{\infty}(\Omega)}$. This is achieved by comparing the relative entropy $\mathrm{e}_{\psi_{p}}\left[u_{1} \mid u_{2}\right]$ with $\left\|u_{1}\right\|_{L^{p}(\Omega)}$ as follows.

Lemma 1.1. Assume that $\Omega$ is a domain in $\mathbb{R}^{d}$. Let $u_{1} \in L_{+}^{1} \cap L^{p}(\Omega)$ with $p \in[1,2]$, $u_{2} \in L_{+}^{1} \cap L^{\infty}(\Omega)$. Then

$$
\left\|u_{2}\right\|_{L^{p}(\Omega)}-\left\|u_{2}\right\|_{L^{p}(\Omega)}^{1-p / 2} \frac{2^{1 / p}}{\sqrt{A}}\left(\left\|u_{2}\right\|_{L^{\infty}(\Omega)}^{p-1} e_{\psi_{p}}\left[u_{1} \mid u_{2}\right]\right)^{1 / 2} \leq\left\|u_{1}\right\|_{L^{p}(\Omega)}
$$

and

$$
\left\|u_{1}\right\|_{L^{p}(\Omega)} \leq\left\|u_{2}\right\|_{L^{p}(\Omega)} f^{-1}\left(\frac{2^{2 / p}}{A} \frac{\left\|u_{2}\right\|_{L^{\infty}(\Omega)}^{p-1} e_{\psi_{p}}\left[u_{1} \mid u_{2}\right]}{\left\|u_{2}\right\|_{L^{p}(\Omega)}^{p}}\right),
$$

where $f$ is the function defined on $[1,+\infty)$ by $f(x)=x^{p-2}(x-1)^{2}$. As a consequence, for a given function $u_{2} \in L_{+}^{1} \cap L^{\infty}(\Omega)$, there exists a continuous function $\kappa: \mathbb{R}^{+} \rightarrow$ $(0,+\infty)$ such that

$$
\kappa(0)=\lim _{e_{0} \rightarrow 0_{+}} \kappa\left(e_{0}\right) \geq \frac{A}{2^{2 / p}} \frac{\left\|u_{2}\right\|_{L^{p}(\Omega)}^{p-2}}{\left\|u_{2}\right\|_{L^{\infty}(\Omega)}^{p-1}},
$$

and for which, for any $u_{1} \in L_{+}^{1} \cap L^{p}(\Omega)$,

$$
e_{\psi_{p}}\left[u_{1} \mid u_{2}\right] \leq e_{0} \Longrightarrow e_{\psi_{p}}\left[u_{1} \mid u_{2}\right] \geq \kappa\left(e_{0}\right)\left\|u_{1}-u_{2}\right\|_{L^{p}(\Omega)}^{2} .
$$

Proof. If $\left\|u_{1}\right\|_{L^{p}(\Omega)}>\left\|u_{2}\right\|_{L^{p}(\Omega)}$, then (1.10) is obvious. Otherwise,

$$
\mathrm{e}_{\psi_{p}}\left[u_{1} \mid u_{2}\right] \geq \frac{A}{2^{2 / p}} \frac{\left\|u_{2}\right\|_{L^{p}(\Omega)}^{p-2}}{\left\|u_{2}\right\|_{L^{\infty}(\Omega)}^{p-1}}\left(\left\|u_{2}\right\|_{L^{p}(\Omega)}-\left\|u_{1}\right\|_{L^{p}(\Omega)}\right)^{2},
$$

which proves (1.10). In both cases,

$$
\frac{2^{2 / p}}{A}\left\|u_{2}\right\|_{L^{\infty}(\Omega)}^{p-1} \mathrm{e}_{\psi_{p}}\left[u_{1} \mid u_{2}\right] \geq \frac{\left(\left\|u_{1}\right\|_{L^{p}(\Omega)}-\left\|u_{2}\right\|_{L^{p}(\Omega)}\right)^{2}}{\left\|u_{1}\right\|_{L^{p}(\Omega)}^{2-p}}=\left\|u_{2}\right\|_{L^{p}(\Omega)}^{p} f\left(\frac{\left\|u_{1}\right\|_{L^{p}(\Omega)}}{\left\|u_{2}\right\|_{L^{p}(\Omega)}}\right),
$$

from which (1.11) easily follows. Assertion (1.12) then follows from Corollary 1.3. 
The next step is to compare $\mathrm{e}_{\psi_{p}}$ with $\mathrm{e}_{\psi_{2}}$, or, to be precise, $\mathrm{I}_{\psi_{p}} / \mathrm{e}_{\psi_{p}}$ with $\mathrm{I}_{\psi_{2}} / \mathrm{e}_{\psi_{2}}$. Since, for $p \in[1,2]$,

$$
\mathrm{I}_{p}[u]=\frac{4}{p} \int_{\Omega}|\nabla w|^{2}|x|^{\alpha} u_{\infty} d x \quad \text { with } \quad v:=\frac{u}{u_{\infty}}, \quad w:=v^{p / 2},
$$

it is convenient to write

$$
\mathrm{e}_{\psi_{p}}\left[u_{1} \mid u_{2}\right]=\int_{\Omega} f_{p}(w) u_{2} d x
$$

with

$$
\begin{array}{ll}
f_{p}(w):=\frac{w^{2}-1-p\left(w^{2 / p}-1\right)}{p-1} & \text { if } \quad p \in(1,2], \\
f_{1}(w):=w^{2} \log w^{2}-\left(w^{2}-1\right) & \text { if } \quad p=1 .
\end{array}
$$

Lemma 1.2. For any $w \in \mathbb{R}^{+}$, for any $p \in(1,2]$,

$$
f_{2}(w) \leq f_{p}(w) \leq \frac{1}{p-1} f_{2}(w) .
$$

If $p=1, f_{1}(w) \geq f_{2}(w)$ for any $w \in \mathbb{R}^{+}$.

Proof. Assume first that $p>1$. From

$$
\frac{d^{2}}{d w^{2}}\left[f_{p}(w)-f_{2}(w)\right]=\frac{2(2-p)}{p-1}\left(1-\frac{1}{p} w^{-\frac{2(p-1)}{p}}\right),
$$

it follows that $w \mapsto f_{p}(w)-f_{2}(w)$ is concave on $(0, w(p))$ and convex on $(w(p), \infty)$, with

$$
w(p)=p^{-\frac{p}{2(p-1)}}<1 \quad \forall p \in(1,2) .
$$

Moreover, $f_{p}^{\prime}(1)-f_{2}^{\prime}(1)=0$ so that for any $w \in \mathbb{R}^{+}$,

$$
f_{p}(w)-f_{2}(w) \geq \inf _{w \in \mathbb{R}^{+}}\left[f_{p}(w)-f_{2}(w)\right]=0=f_{p}(0)-f_{2}(0)=f_{p}(1)-f_{2}(1) .
$$

This proves the lower bound.

As for the upper bound, we simply notice that

$$
0=(p-1) f_{p}(1)-f_{2}(1)=(p-1) f_{p}^{\prime}(1)-f_{2}^{\prime}(1)
$$

and

$$
\frac{d^{2}}{d w^{2}}\left[(p-1) f_{p}(w)-f_{2}(w)\right]=-\frac{2(2-p)}{p} w^{-\frac{2(p-1)}{p}} \leq 0 \quad \forall w \in \mathbb{R}^{+} .
$$

If $p=1$, we can easily check that $f_{1}^{\prime}(1)-f_{2}^{\prime}(1)=0, f_{1}(0)-f_{2}(0)=0$, and $f_{1}^{\prime \prime}(w)-f_{2}^{\prime \prime}(w)$ changes sign only once, as in the case $p>1$.

If we are interested in the direct comparison of $\mathrm{e}_{\psi_{p}}$ with $\mathrm{e}_{\psi_{2}}$, it is simpler to work with $v=u_{1} / u_{2}$. The function $f(v):=|v-1|^{2}-\frac{1}{p-1}\left(v^{p}-1-p(v-1)\right)$ is such that $f(0)=f(1)=f^{\prime}(1)=0, f^{\prime \prime}$ changes sign once on $\mathbb{R}^{2}$, and in the end, $f(v) \geq 0$ for any $v \geq 0$. Thus we have proved the following result. 
Lemma 1.3. Assume that $\Omega$ is a domain in $\mathbb{R}^{d}$. For any $p \in[1,2]$, if $u_{1}$ and $u_{2}$ belong to $L_{+}^{1} \cap L^{2}(\Omega)$ and if $u_{2}>0$ a.e. in $\Omega$, then

$$
e_{\psi_{p}}\left[u_{1} \mid u_{2}\right] \leq e_{\psi_{2}}\left[u_{1} \mid u_{2}\right]
$$

Notations and conventions. For simplicity, if $\psi=\psi_{p}$, we shall denote the entropy $\mathrm{e}_{\psi_{p}}\left[\cdot \mid u_{\infty}\right]$ by

$$
\Sigma_{p}[u]:=\mathrm{e}_{\psi_{p}}\left[u \mid u_{\infty}\right]=\int_{\Omega} \psi_{p}\left(\frac{u}{u_{\infty}}\right) u_{\infty} d x
$$

and the corresponding entropy production term by

$$
\mathrm{I}_{p}[u]:=\int_{\Omega} \psi_{p}^{\prime \prime}\left(\frac{u}{u_{\infty}}\right)\left|\nabla\left(\frac{u}{u_{\infty}}\right)\right|^{2} \sigma u_{\infty} d x .
$$

Here we assume that $u_{\infty}$ is a stationary solution of (1.1), and that it is unique, bounded in $L^{1}(\Omega)$ and normalized such that $\int_{\Omega} u_{\infty} d x=\int_{\Omega} u d x$. As already mentioned at the beginning of this section, if $p=2$, sign changing solutions can be considered, but otherwise for $p \in[1,2)$, the functional $\Sigma_{p}$ is convex only for nonnegative functions $u$. We shall therefore assume from now on that

$$
u \geq 0 \quad(x, t) \in \Omega \times \mathbb{R}^{+} \text {a.e. }
$$

without further notice. Whenever the uniqueness holds (see Section 1.2), any weak solution can always be approximated by a regularized solution. Inequality (1.6) then means that

$$
\frac{d}{d t} \Sigma_{p}[u(\cdot, t)] \leq-\mathrm{I}_{p}[u(\cdot, t)]
$$

at least in the distribution sense with respect to $t$.

To simplify the presentation, using an appropriate rescaling, we can assume that $|\Omega|=1$ if $\Omega$ is bounded. Since equation (2.1) is linear, we can always multiply $u_{0}$ by a constant. If $\int_{\Omega} u_{0} d x \neq 0$, we may therefore assume that $\int_{\Omega} u_{0} d x=1$ so that $\int_{\Omega} u(\cdot, t) d x=1$ for any $t \geq 0$.

\section{Degenerate diffusions}

In this section, we investigate what happens when the diffusion coefficient degenerates at some point inside the domain $\Omega$. This corresponds to a non uniformly elliptic right hand side in Equation (1.1). To avoid technicalities, we focus here to a very special form of Equation (1.1) and refer to Section 4 for examples which are more realistic in view of the applications. 


\subsection{Reformulation of the problem and notations}

As a special case of Equation (1.1), consider the equation

$$
u_{t}=\nabla \cdot\left(|x|^{\alpha} \nabla u+\beta x u\right), \quad \forall(x, t) \in \Omega \times \mathbb{R}^{+}
$$

where $\alpha$ and $\beta$ are positive constants. Assume that $u$ satisfies the initial condition

$$
\left.u\right|_{t=0}=u_{0} \in L_{+}^{1}(\Omega)
$$

and the boundary condition

$$
\nu(x) \cdot\left(|x|^{\alpha} \nabla u+\beta x u\right)=0, \quad \forall(x, t) \in \partial \Omega \times \mathbb{R}^{+} .
$$

Here $\Omega$ is a bounded or unbounded domain in $\mathbb{R}^{d}$ with a smooth boundary such that $0 \in \Omega$, and $\Omega$ is possibly unbounded. Under decay assumptions at infinity if $\Omega$ is unbounded, a smooth nonnegative solution of (2.1) conserves mass:

$$
\frac{d}{d t} \int_{\Omega} u(x, t) d x=0
$$

Let $M=\int_{\Omega} u_{0}(x) d x>0$ and define the zero-flux solution by

$$
\begin{cases}u_{\infty}(x)=\rho e^{\frac{\beta}{\alpha-2}|x|^{2-\alpha}} & \text { if } \quad \alpha \neq 2, \\ u_{\infty}(x)=\frac{\rho}{|x|^{\beta}} & \text { if } \quad \alpha=2 .\end{cases}
$$

Note that both $(2.1)$ and $(2.3)$ are satisfied by $u_{\infty}$. We assume next that $u_{\infty}$ is integrable, which means $\alpha \in(0,2)$ or $\alpha=2$ and $\beta \in(0, d)$. The constant $\rho$ is therefore chosen such that $\int_{\Omega} u_{\infty} d x=M$ :

1) $\rho=M\left(\int_{\Omega} e^{\frac{\beta}{\alpha-2}|x|^{2-\alpha}} d x\right)^{-1}$ if $0 \leq \alpha<2$,

2) $\rho=M\left(\int_{\Omega}|x|^{-\beta} d x\right)^{-1}$ if $\alpha=2, \beta<d$ and $\Omega$ is bounded.

With $v:=u / u_{\infty},(2.1)$ can be rewritten as

$$
u_{\infty} \frac{\partial v}{\partial t}=\nabla \cdot\left(\sigma|x|^{\alpha} u_{\infty} \nabla v\right)
$$

and the boundary conditions (1.2) are equivalent to homogeneous Neumann boundary conditions for $v$ :

$$
\nu \cdot \nabla v=0, \quad \forall(x, t) \in \partial \Omega \times \mathbb{R}^{+} .
$$

Assume that $u_{\infty}$ is integrable and define the relative entropy as in Section 1 . With these notations, the entropy and the entropy production terms are respectively

$$
\mathrm{e}_{\psi}\left[u \mid u_{\infty}\right]=\int_{\Omega} \psi(v) u_{\infty} d x
$$

and, for any convex function $\psi$,

$$
\frac{d}{d t} \mathrm{e}_{\psi}\left[u \mid u_{\infty}\right]=-\int_{\Omega} \psi^{\prime \prime}(v)|x|^{\alpha} u_{\infty}|\nabla v|^{2} d x:=-\mathrm{I}_{\psi}\left[u \mid u_{\infty}\right] \leq 0 .
$$


The convexity property of the function $\psi$ is essential for our approach. As above, for $\psi=\psi_{p}, p \in[1,2]$, we will simply denote these quantities by $\Sigma_{p}[u]$ and $\mathrm{I}_{p}[u]$.

\subsection{On convex entropies}

For $p \in[1,2]$, if we write

$$
v:=\frac{u}{u_{\infty}}, \quad w:=v^{p / 2}, \quad \mathrm{I}_{p}[u]=\frac{4}{p} \int_{\Omega}|\nabla w|^{2}|x|^{\alpha} u_{\infty} d x
$$

the dependence of $\mathrm{I}_{p}$ in $p$ becomes trivial. Let us define a natural set for the functional $\Sigma_{p}$ :

$$
X_{p}(\Omega):=\left\{u \in L_{+}^{1} \cap L^{p}(\Omega): \Sigma_{p}[u]<\infty\right\} .
$$

On $X_{p}(\Omega) \ni u$, we can write $\Sigma_{p}[u]=\int_{\Omega} f_{p}(w) u_{\infty} d x$ where $f_{p}$ is given by $(1.13)$. As a simple consequence of Lemma 1.2, we have as in ${ }^{4}$ the following

Corollary 2.1. For any $p \in(1,2]$,

$$
\frac{2}{p}(p-1) \inf _{u \in X_{p}(\Omega)} \frac{I_{2}[u]}{\Sigma_{2}[u]} \leq \inf _{u \in X_{p}(\Omega)} \frac{I_{p}[u]}{\Sigma_{p}[u]} \leq \frac{2}{p} \inf _{u \in X_{p}(\Omega)} \frac{I_{2}[u]}{\Sigma_{2}[u]} .
$$

\subsection{Case $\alpha \in[0,2)$ : Hardy-Poincaré inequality}

The following Hardy-Poincaré inequality is the crucial tool for proving the exponential decay of the entropy $\mathrm{e}_{\psi_{p}}$. Notice that the limiting case $\alpha=2$ in this inequality is not relevant for Equation (2.1), but is covered by our result.

Theorem 2.1. Consider a bounded connected domain $\Omega \ni 0$. Let $u_{\infty} \in L^{\infty}(\Omega)$ be such that $\int_{\Omega} u_{\infty} d x=1$ and assume that there exist two positive constants $C_{1}$ and $C_{2}$ such that

$$
C_{1} \leq u_{\infty} \leq C_{2} \quad \text { a.e. }
$$

Assume moreover that $\alpha \in[0,2]$ if $d \geq 2$ and $\alpha \in[1,2]$ if $d=1$. Then there exists a positive constant $\lambda_{2}$ such that

$$
\frac{\int_{\Omega}|x|^{\alpha}|\nabla v|^{2} u_{\infty} d x}{\int_{\Omega}|v-1|^{2} u_{\infty} d x} \geq \lambda_{2}
$$

for any $v \in C^{\infty}(\Omega)$ such that $\int_{\Omega} u_{\infty} v d x=1$.

By density, such an inequality also holds in the larger set of functions defined as the completion of $C^{\infty}(\Omega)$ with respect to the norm $\|v\|^{2}=\int_{\Omega}|x|^{\alpha}|\nabla v|^{2} u_{\infty} d x+$ $\int_{\Omega}|v|^{2} u_{\infty} d x$. Note indeed that the average with respect to the measure $u_{\infty} d x$ is well defined because of the Cauchy-Schwarz inequality

$$
\int_{\Omega} v u_{\infty} d x \leq\left(\int_{\Omega} u_{\infty}|v|^{2} d x\right)^{1 / 2}\left(\int_{\Omega} u_{\infty} d x\right)^{1 / 2}
$$


The condition $\int_{\Omega} u_{\infty} d x=1$ is not restrictive because of the homogeneity of the quotient. We denote by $\lambda_{2}$ the best constant, since it is the second eigenvalue of the operator $-u_{\infty}^{-1} \nabla\left(u_{\infty}|x|^{\alpha} \nabla \cdot\right)$ in $L^{2}\left(\Omega, u_{\infty} d x\right)$. The first eigenvalue is $\lambda_{1}=0$, which corresponds to constant eigenfunctions. Homogeneous Neumann boundary conditions are the natural boundary conditions.

Proof. Compared to the standard Hardy-Poincaré inequality, there are two difficulties:

1) We are dealing with the measure $u_{\infty} d x$ and the mean value of $v$ is taken with respect to this measure. This difficulty is considered in Step 1.

2) The domain $\Omega$ is bounded and Neumann boundary conditions have to be considered.

Step 1. We reduce the problem to the case $u_{\infty}=1$. By homogeneity,

$$
\inf _{\substack{u_{\infty} v d x=1 \\ v \neq \equiv 1}} \frac{\int_{\Omega}|x|^{\alpha}|\nabla v|^{2} u_{\infty} d x}{\int_{\Omega}|v-1|^{2} u_{\infty} d x}=\inf _{\nabla v \neq 0} \frac{\int_{\Omega}|x|^{\alpha}|\nabla v|^{2} u_{\infty} d x}{\int_{\Omega}\left|v-\int_{\Omega} v u_{\infty} d x\right|^{2} u_{\infty} d x} .
$$

Adding and substracting $\int_{\Omega} v u_{\infty} d x$ and expanding the square, we get

$$
\begin{gathered}
\int_{\Omega}\left|v-\int_{\Omega} v d x\right|^{2} u_{\infty} d x \\
=\int_{\Omega}\left|v-\int_{\Omega} v u_{\infty} d x+\int_{\Omega} v u_{\infty} d x-\int_{\Omega} v d x\right|^{2} u_{\infty} d x \\
=\int_{\Omega}\left|v-\int_{\Omega} v u_{\infty} d x\right|^{2} u_{\infty} d x+\left[\int_{\Omega} v u_{\infty} d x-\int_{\Omega} v d x\right]^{2} \\
\geq \int_{\Omega}\left|v-\int_{\Omega} v u_{\infty} d x\right|^{2} u_{\infty} d x
\end{gathered}
$$

Thus

$$
\inf _{\nabla v \neq 0} \frac{\int_{\Omega}|x|^{\alpha}|\nabla v|^{2} u_{\infty} d x}{\int_{\Omega}\left|v-\int_{\Omega} v u_{\infty} d x\right|^{2} u_{\infty} d x} \geq \inf _{\nabla v \neq 0} \frac{\int_{\Omega}|x|^{\alpha}|\nabla v|^{2} u_{\infty} d x}{\int_{\Omega}\left|v-\int_{\Omega} v d x\right|^{2} u_{\infty} d x} .
$$

Using the upper and lower bounds on $u_{\infty}$, it follows that

$$
\inf _{\nabla v \neq 0} \frac{\int_{\Omega}|x|^{\alpha}|\nabla v|^{2} u_{\infty} d x}{\int_{\Omega}\left|v-\int_{\Omega} v d x\right|^{2} u_{\infty} d x} \geq \frac{C_{1}}{C_{2}} \inf _{\nabla v \neq 0} \frac{\int_{\Omega}|x|^{\alpha}|\nabla v|^{2} d x}{\int_{\Omega}\left|v-\int_{\Omega} v d x\right|^{2} d x} .
$$

Step 2. Next we have to prove that the right hand side of the above inequality is bounded from below. For any bounded domain $\Omega \subset \mathbb{R}^{d}$, define

$$
\Lambda(\Omega):=\inf _{u \in H^{1}(\Omega)} \frac{\int_{\Omega}|\nabla u|^{2}|x|^{\alpha} d x}{\int_{\Omega}|u|^{2} d x} .
$$


It is elementary to check that $\Lambda(\Omega)$ is positive in one of the two following cases:

(i) $0 \notin \Omega$ and $\Omega$ is connected,

(ii) $\Omega=B(0, R)$ for some $R>0$ and $\alpha \leq 2$.

In the first case $\Lambda(\Omega)$ can be estimated from below by $|d(0, \Omega)|^{\alpha} \lambda_{2}(-\Delta)$ where $\lambda_{2}(-\Delta)$ is the second eigenvalue of the operator $-\Delta$, with homogeneous Neumann boundary conditions.

The case (ii) is easily obtained as follows, if $\alpha<2$. Using a spherical harmonics expansion, it is immediate to see that $\Lambda(\Omega)=\min \left(\lambda_{1}\left(-\Delta, S^{d-1}\right), \lambda_{2}^{r}\right)$, where $\lambda_{1}\left(-\Delta, S^{d-1}\right)$ is the first eigenvalue of the Laplace-Beltrami operator on $S^{d-1}$ and $\lambda_{2}^{r}$ is the first nonzero eigenvalue of the radial problem

$$
-r^{\alpha} \varphi^{\prime \prime}-(d+\alpha-1) r^{\alpha-1} \varphi^{\prime}=\lambda_{2}^{r} \varphi .
$$

Assume first that $\alpha<1$ and define $\rho$ as the first zero of $\psi^{\prime}$ in $(0,+\infty)$, where $\psi$ is the solution of the ODE

$$
-r^{\alpha} \psi^{\prime \prime}-(d+\alpha-1) r^{\alpha-1} \psi^{\prime}=\psi, \quad \psi(0)=1, \quad \psi^{\prime}(0)=0 .
$$

Then, up to a multiplication by a constant, $\varphi(r)=\psi(r \rho / R)$ and $\lambda_{2}^{r}=(\rho / R)^{\alpha-2}>0$. If $\alpha \geq 1$, the condition $\psi^{\prime}(0)=0$ does not make sense, but for $\alpha \in[1,2)$, we may simply replace it by the condition

$$
\lim _{r \rightarrow 0_{+}} r^{\alpha-1} \psi^{\prime}(r)=-\frac{1}{d} .
$$

In the case $\alpha=2$, let $u$ be a function in $C^{1}(0, R)$, with $u(R)=0$. By expanding the square and using an integration by parts for the cross term, we get

$$
0 \leq \int_{0}^{R}\left|r u^{\prime}+\frac{d}{2} u\right|^{2} r^{d-1} d r=\int_{0}^{R} r^{2}\left|u^{\prime}\right|^{2} r^{d-1} d r-\frac{d^{2}}{4} \int_{0}^{R}|u|^{2} r^{d-1} d r,
$$

which means

$$
\int_{\Omega}|u|^{2} d x \leq \frac{4}{d^{2}} \int_{\Omega}|x|^{2}|\nabla u|^{2} d x
$$

if $\Omega=B(0, R)$. Here we write $u(x)=u(|x|)$ which is a standard abuse of notations. Since

$$
\int_{\Omega}|u-\bar{u}|^{2} d x=\int_{\Omega}|u|^{2} d x-|\Omega| \bar{u}^{2} \leq \int_{\Omega}|u|^{2} d x,
$$

we conclude that

$$
\int_{\Omega}|u-\bar{u}|^{2} d x \leq \frac{4}{d^{2}} \int_{\Omega}|x|^{2}|\nabla u|^{2} d x .
$$

Note that the optimal constant in the above inequality is realized by radial functions, as can be shown by using Schwarz' symmetrization method in the ball in $\mathbb{R}^{d+2}$. Of course, we may now shift $u$ by any arbitrary constant, so that the condition $u(R)=0$ can be removed. This proves that for $\alpha=2, \Lambda(\Omega) \geq d^{2} / 4$. 
Notice that for $\alpha>2$, any minimizing sequence concentrates and $\Lambda(\Omega)=0$, so that the restriction $\alpha \in[0,2]$ makes sense.

We have now to extend the results corresponding to the special cases (i) and (ii) to a general bounded connected domain. This is done using the next lemma, which completes the proof of Theorem 2.1.

Lemma 2.1. Let $\Omega$ be a bounded connected open set in $\mathbb{R}^{d}$ such that $0 \in \Omega$. If $\alpha \in[0,2]$, then $\Lambda(\Omega)$ is positive.

Proof. Let $R>0$ be such that $B(0,3 R) \subset \Omega$ and $\Omega \backslash B(0,3 R)$ is connected. Define

$$
\Omega_{1}:=B(0,2 R), \quad \Omega_{2}:=\Omega \backslash B(0,2 R) \quad \text { and } \quad \Omega_{3}:=B(0,3 R) \backslash B(0, R) .
$$

As seen above, $\Lambda\left(\Omega_{i}\right)>0$ for $i=1,2,3$. Assume by contradiction that $\Lambda(\Omega)=0$ and consider a sequence $\left(u_{n}\right)_{n \in \mathbb{N}}$ of functions in $H^{1}(\Omega)$ such that

$$
\int_{\Omega} u_{n} d x=0, \quad \int_{\Omega}\left|u_{n}\right|^{2} d x=1 \quad \text { and } \quad \lim _{n \rightarrow \infty} \int_{\Omega}\left|\nabla u_{n}\right|^{2}|x|^{\alpha} d x=0 .
$$

Let $a_{n}:=\int_{\Omega_{1}} u_{n} d x$ and notice that $a_{n}:=-\int_{\Omega_{2}} u_{n} d x$. Since $\left(a_{n}\right)_{n \in \mathbb{N}}$ is uniformly bounded, up to the extraction of a subsequence we may assume that it converges to some limit $a$. Also define

$$
x_{n}:=\int_{\Omega_{1}}\left|\nabla u_{n}\right|^{2}|x|^{\alpha} d x, y_{n}:=\int_{\Omega_{2}}\left|\nabla u_{n}\right|^{2}|x|^{\alpha} d x \text { and } z_{n}:=\int_{\Omega_{3}}\left|\nabla u_{n}\right|^{2}|x|^{\alpha} d x .
$$

By assumption, $\lim _{n \rightarrow \infty} x_{n}=\lim _{n \rightarrow \infty} y_{n}=\lim _{n \rightarrow \infty} z_{n}=0$. On the other hand, by definition of $\Lambda\left(\Omega_{1}\right)$ and $\Lambda\left(\Omega_{2}\right)$,

$$
\int_{\Omega_{1}}\left|u_{n}-\frac{a_{n}}{\left|\Omega_{1}\right|}\right|^{2} d x \leq \frac{x_{n}}{\Lambda\left(\Omega_{1}\right)} \quad \text { and } \quad \int_{\Omega_{2}}\left|u_{n}+\frac{a_{n}}{\left|\Omega_{2}\right|}\right|^{2} d x \leq \frac{y_{n}}{\Lambda\left(\Omega_{2}\right)},
$$

so that $u_{n}$ converges almost everywhere to $\frac{a}{\left|\Omega_{1}\right|}$ on $\Omega_{1}$ and to $-\frac{a}{\left|\Omega_{2}\right|}$ on $\Omega_{2}$. Let $b_{n}:=\int_{\Omega_{3}} u_{n} d x$ and consider $b:=\lim _{n \rightarrow \infty} b_{n}$, up to the extraction of a further subsequence. By definition of $\Lambda\left(\Omega_{3}\right)$,

$$
\int_{\Omega_{3}}\left|u_{n}-\frac{b_{n}}{\left|\Omega_{3}\right|}\right|^{2} d x \leq \frac{z_{n}}{\Lambda\left(\Omega_{3}\right)}
$$

so that $u_{n}$ converges almost everywhere to $\frac{b}{\left|\Omega_{3}\right|}$ on $\Omega_{3}$. Since $\Omega_{1} \cap \Omega_{3} \neq \emptyset$ and $\Omega_{2} \cap \Omega_{3} \neq \emptyset$, it follows that $\frac{a}{\left|\Omega_{1}\right|}=-\frac{a}{\left|\Omega_{2}\right|}=\frac{b}{\left|\Omega_{3}\right|}$. This proves that $a=0$, a contradiction with

$$
\begin{aligned}
1=\int_{\Omega}\left|u_{n}\right|^{2} d x & =\int_{\Omega_{1}}\left|u_{n}-\frac{a_{n}}{\left|\Omega_{1}\right|}\right|^{2} d x+\int_{\Omega_{2}}\left|u_{n}+\frac{a_{n}}{\left|\Omega_{2}\right|}\right|^{2} d x+a_{n}^{2}\left(\frac{1}{\left|\Omega_{1}\right|}+\frac{1}{\left|\Omega_{2}\right|}\right) \\
& \leq \frac{x_{n}}{\Lambda\left(\Omega_{1}\right)}+\frac{y_{n}}{\Lambda\left(\Omega_{2}\right)}+a_{n}^{2}\left(\frac{1}{\left|\Omega_{1}\right|}+\frac{1}{\left|\Omega_{2}\right|}\right) \rightarrow 0 \quad \text { as } n \rightarrow \infty
\end{aligned}
$$




\subsection{Case $\alpha=2$ : Hardy's inequality}

In the framework of the zero flux solution (2.3) of Equation (2.1), the case $\alpha=2$ has to be treated separately since $u_{\infty}(x)=|x|^{-\beta}$ is not bounded and has to be combined with the weight $|x|^{\alpha}$ to get an estimate of $\Lambda(\Omega)$. The case with Dirichlet boundary conditions or in the whole space has widely treated in the literature, see for instance ${ }^{13,34}$. We are not aware of any result corresponding to Neumann boundary conditions and will therefore consider only the simple case of a ball.

Proposition 2.1. Assume that $\Omega=B(0,1) \subset \mathbb{R}^{d}, d \geq 3$. Let $u_{\infty}$ be as above and assume that $\beta<d$. Then there exists a positive constant $\lambda_{2}$ such that

$$
\frac{\int_{\Omega}|x|^{2-\beta}|\nabla v|^{2} d x}{\int_{\Omega}|v-1|^{2}|x|^{-\beta} d x} \geq \lambda_{2}
$$

for any $v \in C^{\infty}(\Omega)$ such that $\int_{\Omega} u_{\infty} v d x=1$.

As in the case $\alpha<2, \lambda_{2}$ is a second eigenvalue of an operator, namely $-|x|^{\beta} \nabla\left(|x|^{2-\beta} \nabla \cdot\right)$, acting on $L^{2}\left(\Omega,|x|^{-\beta} d x\right)$. The result can also, as in the case of Theorem 2.1, be extended to a larger class of functions by density.

Proof. The result follows from the Caffarelli-Kohn-Nirenberg type inequality

$$
\int_{\Omega} \frac{|\nabla g|^{2}}{|x|^{2 a}} d x \geq C_{\mathrm{CKN}}\left(\int_{\Omega} \frac{g^{q}}{|x|^{b q}} d x\right)^{2 / q}
$$

in the limit case $a=\beta / 2-1, b=\beta / 2, q=2$ after noticing as in the proof of Theorem 2.1 that

$$
\int_{\Omega}\left|v-\int_{\Omega} v d x\right|^{2} u_{\infty} d x \geq \int_{\Omega}\left|v-\int_{\Omega} v u_{\infty} d x\right|^{2} u_{\infty} d x .
$$

This case is a generalized form of Hardy's inequality. The positivity of $C_{\mathrm{CKN}}$ is equivalent to the positivity of the infimum of

$$
\int_{(0, \infty) \times S^{d-1}}\left(|\nabla h|^{2}+\frac{1}{4}(d-2-2 a)^{2}|h|^{2}\right) d s d \omega \cdot\left(\int_{(0, \infty) \times S^{d-1}}|h|^{q} d s d \omega\right)^{-2 / q}
$$

where $h$ is given by $g(x)=|x|^{1+a-d / 2} h(s=-\log |x|, \omega=x /|x|)$ (see $\left.{ }^{13}\right)$ and satisfies the boundary condition $\partial_{s} h(0, \cdot)=0$. By symmetry with respect to $s=0$, the problem is easily reduced to $(s, \omega) \in \mathbb{R} \times S^{d-1}$ which corresponds to the usual Caffarelli-Kohn-Nirenberg inequality.

\subsection{Decay rates}

We can now apply the results of Sections 2.1-2.4 to the study of the asymptotic behaviour of the solutions of (2.1).

Theorem 2.2. Let $\Omega \ni 0$ be a domain with a smooth boundary, $p \in(1,2]$ and consider a weak nonnegative solution $u$ of (2.1)-(2.3) with initial datum $u_{0} \in L^{1} \cap$ 
$L^{p}(\Omega)$. Assume that $\alpha \in[0,2]$. If $\alpha=2$, assume moreover that $\Omega=B(0,1) \subset \mathbb{R}^{d}$ and $\beta<d, d \geq 3$. With the notations of Theorem 2.1 and Proposition 2.1, we have

$$
\Sigma_{p}[u(\cdot, t)] \leq \Sigma_{p}\left[u_{0}\right] e^{-\frac{4}{p}(p-1) \lambda_{2} t} \quad \forall t \geq 0 .
$$

Proof. According to (1.4) and Theorem 2.1 and Proposition 2.1, for a solution of (2.1),

$$
\frac{d}{d t} \Sigma_{p}[u(\cdot, t)]=-\mathrm{I}_{p}[u(\cdot, t)] \leq-\frac{2}{p}(p-1) \cdot 2 \lambda_{2} \Sigma_{p}[u(\cdot, t)] .
$$

The result follows by Gronwall's lemma.

Corollary 2.2. Under the same assumptions as in Theorem 2.2, if $\alpha \in[0,2)$ and if $u_{\infty}$ is given by (2.4), for any solution $u$ of (2.1), there exists a constant $K>0$ which depends only on $\Sigma_{p}\left[u_{0}\right]$ such that

$$
\left\|u(\cdot, t)-u_{\infty}\right\|_{L^{p}(\Omega)} \leq K e^{-\frac{2}{p}(p-1) \lambda_{2} t} \quad \forall t \geq 0 .
$$

Proof. Apply the generalized Csiszár-Kullback inequality (1.9) and notice that since $\Sigma_{p}[u(\cdot, t)]$ is nonnegative, $u(\cdot, t)$ converges to $u_{\infty}$ in $L^{p}(\Omega)$ as $t \rightarrow \infty$, so that, uniformly in $t \geq 0,\|u(\cdot, t)\|_{L^{p}(\Omega)}$ is bounded from below away from 0 by Lemma 1.1.

Note that in case $p=2$, the convergence of $u$ to $u_{\infty}$ in $L^{1}(\Omega)$ is a simple consequence of the Cauchy-Schwarz inequality:

$$
\left\|u-u_{\infty}\right\|_{L^{1}(\Omega)}^{2}=\left(\int_{\Omega}\left|\frac{u}{u_{\infty}}-1\right| \sqrt{u_{\infty}} \cdot \sqrt{u_{\infty}} d x\right)^{2} \leq \Sigma_{2}[u] \cdot \int_{\Omega} u_{\infty} d x=\Sigma_{2}[u] .
$$

If $\Sigma_{2}\left[u_{0}\right]$ is finite, by Lemma 1.3 we get

$$
\Sigma_{p}[u(\cdot, t)] \leq \Sigma_{2}[u(\cdot, t)] \leq \Sigma_{2}\left[u_{0}\right] e^{-2 \lambda_{2} t} \quad \forall t \geq 0,
$$

and then by Lemma 1.1,

$$
\left\|u(\cdot, t)-u_{\infty}\right\|_{L^{p}(\Omega)} \leq K e^{-\lambda_{2} t} \quad \forall t \geq 0,
$$

but in this case $K$ depends on $\Sigma_{2}\left[u_{0}\right]$ and not only on $\Sigma_{p}\left[u_{0}\right]$.

\section{Diffusion equations with time-periodic coefficients}

We are now going to investigate the effect of the time-dependence of the coefficients of the equation in the large time behavior of the solutions. As in Section 2, we will consider a simple case: We will assume that the dependence of the coefficients is time-periodic.

\subsection{Examples}

Before giving a general result, let us start with some examples for which the computations are explicit. In this section, we will focus on time dependent coefficients, mostly time-periodic, and assume that the operator is uniformly elliptic. 


\subsubsection{Example of a drift with time-periodic intensity}

Consider in $\mathbb{R}^{d}$ a solution $u$ of

$$
u_{t}=\Delta u+\beta(t) \nabla(x u), \quad u(\cdot, t=0)=u_{0}
$$

for some function $\beta$ given by

$$
\beta=\gamma R^{2}+\frac{\dot{R}}{R}
$$

Here $\gamma$ is a given positive constant, and we shall assume that $R$ is a periodic function of period $T$ such that there exists two constants $R_{1}>0$ and $R_{2}>0$ for which

$$
0<R_{1}<R(t)<R_{2}<+\infty \quad \forall t \in[0, T] .
$$

Let $R_{0}:=R(0)$ and

$$
u_{\infty}(x, t):=R^{d} \frac{\left\|u_{0}\right\|_{L^{1}\left(\mathbb{R}^{d}\right)}}{(2 \pi)^{d / 2}} e^{-\frac{1}{2} R^{2}|x|^{2}} \quad \text { with } \quad R=R(t) .
$$

Proposition 3.1. Let $d \geq 1$. For $\beta$ and $R$ satisfying (3.2) and (3.3), any solution of (3.1) with nonnegative initial data $u_{0} \in L^{1} \cap L^{p}\left(\mathbb{R}^{d}\right)$ for some $p \in[1,2]$, such that $\left(1+|x|^{2}\right) u_{0} \in L^{1}\left(\mathbb{R}^{d}\right)$, and $u_{0} \log u_{0} \in L^{1}\left(\mathbb{R}^{d}\right)$ if $p=1$, satisfies

$$
\left\|u(\cdot, t)-u_{\infty}(\cdot, t)\right\|_{L^{p}\left(\mathbb{R}^{d}\right)} \leq C_{p} e^{-\gamma \int_{0}^{t} R^{2}(s) d s} \quad \forall t>0,
$$

for some positive constant $C_{p}$ which depends only on $\Sigma_{p}\left[\frac{1}{R_{0}^{d}} u_{0}\left(\frac{\dot{\bar{R}}}{R_{0}}\right)\right]$ and $M$.

Proof. Consider the function $v$ defined by

$$
u(x, t)=R^{d} v(\tau, R x)
$$

where $R=R(t)$ and $\beta=\beta(t)$ are related by (3.2), and where $\tau$ satisfies

$$
\dot{\tau}=R^{2}, \quad \tau(0)=0 .
$$

It is immediate to check that $v$ is a solution of the Fokker-Planck equation

$$
v_{\tau}=\Delta v+\gamma \nabla(x v), \quad v(\cdot, \tau=0)=R_{0}^{-d} u_{0}\left(\cdot / R_{0}\right)=: v_{0},
$$

which converges to

$$
v_{\infty}(x)=\frac{\left\|u_{0}\right\|_{L^{1}\left(\mathbb{R}^{d}\right)}}{(2 \pi)^{d / 2}} e^{-\gamma|x|^{2} / 2} .
$$

We can then apply standard results for the Fokker-Planck equation. With the same notations as in Section 2, i.e., $\Sigma_{p}[v]=\mathrm{e}_{\psi_{p}}\left[v \mid v_{\infty}\right]$ and

$$
\mathrm{I}_{p}[v]:=\int_{\Omega} \psi_{p}^{\prime \prime}\left(\frac{v}{v_{\infty}}\right)\left|\nabla\left(\frac{v}{v_{\infty}}\right)\right|^{2} v_{\infty} d x
$$

we have

$$
\frac{d}{d \tau} \Sigma_{p}[v(\cdot, \tau)]=-\mathrm{I}_{p}[v(\cdot, \tau)]
$$


By the convex Sobolev inequalities (or generalized Poincaré inequalities: see Section 5 for more details), we have $\Sigma_{p}[v(\cdot, \tau)] \leq(2 \gamma)^{-1} \mathrm{I}_{p}[v(\cdot, \tau)]$ and

$$
\frac{d}{d \tau} \Sigma_{p}[v(\cdot, \tau)] \leq-2 \gamma \Sigma_{p}[v(\cdot, \tau)] .
$$

This means that

$$
\Sigma_{p}[v(\cdot, \tau)] \leq \Sigma_{p}\left[v_{0}\right] e^{-2 \gamma \tau},
$$

which controls $\left\|u(\cdot, t)-u_{\infty}(\cdot, t)\right\|_{L^{p}\left(\mathbb{R}^{d}\right)}^{2}$ by (1.9), (1.10) and (1.11). This proves (3.4) by undoing the change of variables.

If $p=1, C_{1}^{2}=4\left\|v_{0}\right\|_{L^{1}\left(\mathbb{R}^{d}\right)} \Sigma_{1}\left[v_{0}\right]$. If $p=2, \Sigma_{2}[v]=\left\|v-v_{\infty}\right\|_{L^{2}\left(\mathbb{R}^{d}\right)}^{2}$ and $C_{2}^{2}=R_{2}^{d}$. Otherwise, for $p \in(1,2),(1.9)$ involves $\|v(\cdot, \tau)\|_{L^{p}\left(\mathbb{R}^{d}\right)}$, which is however bounded uniformly in $\tau$ by Lemma 1.1.

To illustrate the relation between $R$ and $\beta$, we give two explicit examples:

(i) If $R(t)=2+\cos t$, then $\beta(t)=\gamma(2+\cos t)^{2}+\frac{\sin t}{2+\cos t}$.

(ii) Let $\gamma \in(0,1], \theta \in[0,1)$ and consider the 1-periodic function

$$
\left\{\begin{array}{lll}
\beta(t)=0 & \text { if } & 0 \leq t<\theta, \\
\beta(t)=1 & \text { if } & \theta \leq t<1 .
\end{array}\right.
$$

Then $R$ given by (3.2) is periodic if and only if

$$
2 \gamma \theta=(\gamma-1)\left(1-e^{2(1-\theta)}\right) \Longleftrightarrow \gamma=\frac{e^{2(1-\theta)}-1}{e^{2(1-\theta)}-1+2 \theta}
$$

and

$$
\left\{\begin{array}{lll}
R(t)=(1+2 \gamma t)^{-1 / 2} & \text { if } & 0 \leq t<\theta \\
R(t)=\left(1+(\gamma-1)\left(1-e^{-2(t-1)}\right)\right)^{-1 / 2} & \text { if } & \theta \leq t<1
\end{array}\right.
$$

\subsubsection{Example of a drift centered at a time-periodic point}

Consider in $\mathbb{R}^{d}$ a solution $u$ of

$$
u_{t}=\Delta u+\nabla[(x-y(t)) u], \quad u(\cdot, t=0)=u_{0}
$$

for some given function $t \mapsto y(t)$ which takes its values in $\mathbb{R}^{d}$. Let $z$ be the solution of

$$
\dot{z}=y-z, \quad z(0)=z_{0} \in \mathbb{R}^{d},
$$

which can be written as

$$
z(t)=z_{0} e^{-t}+\int_{0}^{t} e^{-(t-s)} y(s) d s .
$$

We shall assume that

$y$ is a periodic function of period $T$ 
and choose

$$
z_{0}=\frac{\int_{0}^{T} e^{s} y(s) d s}{e^{T}-1}
$$

so that $t \mapsto z(t)$ is also a periodic function of period $T$. Consider

$$
u_{\infty}(x, t)=\frac{\left\|u_{0}\right\|_{L^{1}\left(\mathbb{R}^{d}\right)}}{(2 \pi)^{d / 2}} e^{-|x-z(t)|^{2} / 2} .
$$

Proposition 3.2. Let $d \geq 1$. Under the above assumptions, any solution of (3.5) with nonnegative initial data $u_{0} \in L^{1} \cap L^{p}\left(\mathbb{R}^{d}\right)$ for some $p \in[1,2]$, such that $(1+$ $\left.|x|^{2}\right) u_{0} \in L^{1}\left(\mathbb{R}^{d}\right)$ and $u_{0} \log u_{0} \in L^{1}\left(\mathbb{R}^{d}\right)$ if $p=1$, satisfies

$$
\left\|u(\cdot, t)-u_{\infty}(\cdot, t)\right\|_{L^{p}\left(\mathbb{R}^{d}\right)} \leq C_{p} e^{-t} \quad \forall t>0,
$$

for some positive constant $C_{p}$ which depends only on $\Sigma_{p}\left[u_{0}\left(\cdot+z_{0}\right)\right]$ and $\left\|u_{0}\right\|_{L^{1}\left(\mathbb{R}^{d}\right)}$.

Proof. The function

$$
v(x, t)=u(t, x+z(t))
$$

is a solution of the Fokker-Planck equation

$$
v_{t}=\Delta v+\nabla(x v), \quad v(\cdot, \tau=0)=u_{0}\left(\cdot+z_{0}\right),
$$

which converges to

$$
v_{\infty}(x)=\frac{\left\|u_{0}\right\|_{L^{1}\left(\mathbb{R}^{d}\right)}}{(2 \pi)^{d / 2}} e^{-|x|^{2} / 2}
$$

as in the proof of Proposition 3.1. By $\Sigma_{p}\left[u_{0}\left(\cdot+z_{0}\right)\right]$ in (3.6), we mean $\mathrm{e}_{\psi_{p}}\left[u_{0}(\cdot+\right.$ $\left.\left.z_{0}\right) \mid v_{\infty}\right]$.

\subsubsection{Example of diffusions with a general time-periodic drift term}

If the drift term $\psi$ in the equation

$$
u_{t}=\Delta u+\nabla[u \nabla \psi] \quad \forall(x, t) \in \mathbb{R}^{d} \times \mathbb{R}^{+}
$$

with initial data $u(\cdot, t=0)=u_{0}$, does not depend on the time, the asymptotic state is given as the Gibbs state:

$$
u_{\infty}:=\frac{1}{Z}\left\|u_{0}\right\|_{L^{1}\left(\mathbb{R}^{d}\right)} e^{-\psi} \quad \text { with } Z=\int_{\mathbb{R}^{d}} e^{-\psi} d x .
$$

As seen in the two previous examples, the time-dependence of the drift term is reflected by a delay and the Gibbs state is not any more a solution. This is a general feature of diffusion equations with time-periodic coefficients that can be understood as follows. Consider in $\mathbb{R}^{d}$ two functions $\varphi$ and $\psi$ in $C^{2}\left(\mathbb{R} \times \mathbb{R}^{d}\right)$ such that

$$
\begin{aligned}
& \varphi_{t}+\frac{\dot{Z}}{Z}-\Delta \varphi+|\nabla \varphi|^{2}+\Delta \psi-\nabla \varphi \cdot \nabla \psi=0 \\
& \text { where } Z(t)=\int_{\mathbb{R}^{d}} e^{-\varphi(x, t)} d x
\end{aligned}
$$


and assume that both

$$
\varphi \text { and } \psi \text { are periodic with (same) period } T \text {. }
$$

The existence of a solution to (3.8) satisfying (3.9) will be established in Section 3.2 under some additional assumptions. Let $u$ be a solution of (3.7) and define

$$
u_{\infty}(x, t)=\frac{1}{Z(t)}\left\|u_{0}\right\|_{L^{1}\left(\mathbb{R}^{d}\right)} e^{-\varphi(x, t)} .
$$

This allows to state the following formal result.

Proposition 3.3. Let $d \geq 1$. Assume that there exists a solution to (3.8) satisfying (3.9). Under the above assumptions, any solution of (3.7) with nonnegative initial data $u_{0} \in L^{1} \cap L^{p}\left(\mathbb{R}^{d}\right)$ for some $p \in[1,2]$, such that $\left(1+\sup _{t \in[0, T]}|\varphi(\cdot, t)|\right) u_{0} \in$ $L^{1}\left(\mathbb{R}^{d}\right)$, and $u_{0} \log u_{0} \in L^{1}\left(\mathbb{R}^{d}\right)$ if $p=1$, converges to $u_{\infty}$ in $L^{\infty}\left((t,+\infty), L^{p}\left(\mathbb{R}^{d}\right)\right)$ as $t \rightarrow+\infty$.

Proof. It is straightforward to check that $u_{\infty}$ is a solution of (3.7) if and only if (3.8) is satisfied. The result is then a consequence of the contraction property stated in Theorem 1.1, Inequality (1.6) for $\psi=\psi_{p}$ and the generalized Csiszár-Kullback inequality, see Proposition 1.1.

Note that if $\psi(x, t)=\frac{1}{2}|x|^{2}+\chi(x, t)$ where $\chi$ is a periodic bounded function, then one can solve (3.8) so that (3.9) holds, and then get an exponential convergence rate using the Holley-Stroock lemma (see Section 5 and ${ }^{5}$ for more details). The generalization to convex quadratic or super-quadratic potentials is easy.

\subsection{Convergence to time-periodic solutions}

Here we state a generalization to any dimension of previous results obtained in ${ }^{20}$ when the dimension is $d=1$. The presentation closely follows the results in ${ }^{20}$.

Consider a solution of the time-dependent drift-diffusion equation

$$
u_{t}=\nabla \cdot[\sigma(x, t) \nabla u+\nabla \phi(x, t) u]
$$

for $t \geq 0, x \in \Omega$, where $\Omega$ is a bounded domain in $\mathbb{R}^{d}, d \geq 1$, such that

$$
\Lambda_{\phi, \sigma}^{(T)}:=\inf _{(x, t) \in \Omega \times[0, T)} \inf _{u} \frac{\mathrm{I}_{\psi_{1}}\left[u \mid u_{\phi, \sigma}\right]}{\mathrm{e}_{\psi_{1}}\left[u \mid u_{\phi, \sigma}\right]}>0,
$$

where

$$
u_{\phi, \sigma}:=\frac{e^{-\phi / \sigma}}{\int_{\Omega} e^{-\phi / \sigma} d x}
$$

is a local (time-dependent) Gibbs state. A sufficient condition for (3.11) to hold is that $\Omega$ is a bounded convex domain if $\phi / \sigma$ is a bounded function. See ${ }^{5}$ for more details. For completeness, we will give a proof based on the entropy - entropy production method in the last section, in the one dimensional case. 
Notice that in general $u_{\phi, \sigma}$ is not a solution of (3.10). Let

$$
h_{\phi, \sigma}(t):=\left\|\frac{\left(u_{\phi, \sigma}\right)_{t}(\cdot, t)}{u_{\phi, \sigma}(\cdot, t)}\right\|_{L^{\infty}(\Omega)} .
$$

Assume that $u$ satisfies Robin (no flux) boundary conditions:

$$
[\sigma(x, t) \nabla u+\nabla \phi(x, t) u] \cdot \nu(x)=0 \quad \forall(x, t) \in \partial \Omega \times \mathbb{R}^{+}
$$

and that $\sigma$ and $\phi$ are time-periodic of period T. Assume moreover for simplicity that $\sigma$ is uniformly positive:

$$
\inf _{(x, t) \in \Omega \times[0, T)} \sigma(x, t)=\sigma_{0}>0 .
$$

These assumptions are mostly motivated by flashing ratchet models.

Theorem 3.1. Let $\phi \in L^{\infty}([0, T) \times \Omega)$ be a T-periodic potential and assume that there exists a finite partition of $[0, T)$ into intervals $\left[T_{i}, T_{i+1}\right), i=0, \ldots, n$ with $T_{0}=0, T_{n}=T$ such that $\left(h_{\phi, \sigma}\right)_{\mid\left[T_{i}, T_{i+1}\right)} \in L^{\infty}\left(T_{i}, T_{i+1}\right)$. Under Assumptions (3.11) and (3.12), there exists a T-periodic nonnegative solution $u_{\infty}$ to (3.10) such that

$$
\left\|u_{\infty}(\cdot, t)\right\|_{L^{1}(\Omega)}=1 \quad \forall t \in[0, T) .
$$

Moreover, there exist a constant $\mathcal{K}_{\phi, \sigma}$ depending on $\phi$ and $\sigma$ only such that:

$$
\sup _{t \in[0, T)}\left\|u_{\infty}(\cdot, t)-u_{\phi, \sigma}(\cdot, t)\right\|_{L^{1}(\Omega)} \leq \mathcal{K}_{\phi, \sigma} .
$$

Proof. For simplicity, we assume first that there are no discontinuities in $t$ and prove the result. Then we show how to extend it in the case of two subintervals of continuity $(n=2)$. The general case is left to the reader.

Step 1. - Assume first that $n=1$ and define $K_{\phi, \sigma}:=\sup _{0 \leq t<T} h_{\phi, \sigma}(t)$. Consider a solution $u$ of $(3.10)$ with $\|u(\cdot, t)\|_{L^{1}(\Omega)}=1$. Then

$$
\frac{d}{d t} \mathrm{e}_{\psi_{1}}\left[u \mid u_{\phi, \sigma}\right]=-\mathrm{I}_{\psi_{1}}\left[u \mid u_{\phi, \sigma}\right]-\int_{\Omega}\left[\frac{u}{u_{\phi, \sigma}}-1\right]\left(u_{\phi, \sigma}\right)_{t} d x .
$$

Using the fact that $\int_{\Omega}\left(u_{\phi, \sigma}\right)_{t} d x=\frac{d}{d t} \int_{\Omega}\left(u_{\phi, \sigma}\right) d x=0$, we can estimate the last term of the right hand side by $K_{\phi, \sigma}$, so that

$$
\frac{d}{d t} \mathrm{e}_{\psi_{1}}\left[u \mid u_{\phi, \sigma}\right] \leq-\mathrm{I}_{\psi_{1}}\left[u \mid u_{\phi, \sigma}\right]+K_{\phi, \sigma} .
$$

A Gronwall estimate shows that

$$
\mathrm{e}_{\psi_{1}}\left[u(\cdot, T) \mid u_{\phi, \sigma}(\cdot, T)\right] \leq \mathrm{e}_{\psi_{1}}\left[u(\cdot, 0) \mid u_{\phi, \sigma}(\cdot, 0)\right] e^{-\Lambda_{\phi, \sigma}^{(T)} T}+\frac{K_{\phi, \sigma}}{\Lambda_{\phi, \sigma}^{(T)}}\left(1-e^{-\Lambda_{\phi, \sigma}^{(T)} T}\right)
$$

so that

$$
\mathrm{e}_{\psi_{1}}\left[u(\cdot, 0) \mid u_{\phi, \sigma}(\cdot, 0)\right] \leq \frac{K_{\phi, \sigma}}{\Lambda_{\phi, \sigma}^{(T)}} \quad \Longrightarrow \quad \mathrm{e}_{\psi_{1}}\left[u(\cdot, T) \mid u_{\phi, \sigma}(\cdot, T)\right] \leq \frac{K_{\phi, \sigma}}{\Lambda_{\phi, \sigma}^{(T)}}
$$


Step 2. - We will now assume that

$$
\phi(x, t)= \begin{cases}\phi_{1}(t, x) & \text { if } \quad 0 \leq t<T_{1} \\ \phi_{2}(t, x) & \text { if } \quad T_{1} \leq t<T_{2} \equiv T\end{cases}
$$

and $\phi$ is periodic with period $T$. We now have to modify the argument from the previous step taking into account the fact that $\phi$, hence $u_{\phi, \sigma}$ is in general not differentiable with respect to $t$ at $t=T_{1}$. Let $u_{1}:=u_{\phi, \sigma \mid t \in\left[0, T_{1}\right)}$ and $u_{2}:=u_{\phi, \sigma \mid t \in\left[T_{1}, T_{2}\right)}$. Using Step 1 we get

$$
\begin{aligned}
& \mathrm{e}_{\psi_{1}}\left[u \mid u_{1}\right]_{\mid t=T_{1}} \leq \mathrm{e}_{\psi_{1}}\left[u \mid u_{1}\right]_{\mid t=0} e^{-\Lambda_{\phi_{1}, \sigma}^{\left(T_{1}\right)} T_{1}}+\frac{K_{\phi_{1}}\left(1-e^{-\Lambda_{\phi_{1}, \sigma}^{\left(T_{1}\right)} T_{1}}\right)}{\Lambda_{\phi_{1}, \sigma}^{\left(T_{1}\right)}} \\
& \mathrm{e}_{\psi_{1}}\left[u \mid u_{2}\right]_{\mid t=T} \leq \mathrm{e}_{\psi_{1}}\left[u \mid u_{2}\right]_{\mid t=T_{1}} e^{-\Lambda_{\phi_{2}, \sigma}^{\left(T_{2}\right)}\left(T_{2}-T_{1}\right)}+\frac{K_{\phi_{2}}\left(1-e^{-\Lambda_{\phi_{2}, \sigma}^{\left(T_{2}\right)}\left(T_{2}-T_{1}\right)}\right)}{\Lambda_{\phi_{2}, \sigma}^{\left(T_{2}\right)}}
\end{aligned}
$$

For each nonnegative function $v$ we have

$$
\mathrm{e}_{\psi_{1}}\left[v \mid u_{i}\right]=\mathrm{e}_{\psi_{1}}[v \mid 1]-\int_{\Omega} u \log u_{\phi, i} d x,
$$

hence from (3.14)-(3.15) we get

$$
\mathrm{e}_{\psi_{1}}[u \mid 1]_{\mid t=T} \leq \mathrm{e}_{\psi_{1}}[u \mid 1]_{\mid t=0} e^{-\Lambda_{\phi_{1}, \sigma}^{\left(T_{1}\right)} T_{1}-\Lambda_{\phi_{2}, \sigma}^{\left(T_{2}\right)}\left(T_{2}-T_{1}\right)}+K\left(\phi_{1}, \phi_{2}, T_{1}, T_{2}\right),
$$

where $K\left(\phi_{1}, \phi_{2}, T_{1}, T_{2}\right)$ is a constant depending on $\phi_{1}, \phi_{2}, T_{1}, T_{2}$ only. Just like in the case of smooth potentials, the set

$$
Y=\left\{u \in H_{+}^{1}(\Omega):\|u\|_{L^{1}(\Omega)}=1, \mathrm{e}_{\psi_{1}}[u \mid 1] \leq \frac{K\left(\phi_{1}, \phi_{2}, T_{1}, T_{2}\right)}{1-e^{-\Lambda_{\phi_{1}, \sigma}^{\left(T_{1}\right)} T_{1}-\Lambda_{\phi_{2}, \sigma}^{\left(T_{2}\right)}\left(T_{2}-T_{1}\right)}}\right\}
$$

is stable under the action of the map $\mathcal{T}: u(\cdot, 0) \mapsto \mathcal{T}(u(\cdot, 0))=u(\cdot, T)$.

Step 3. - The map $\mathcal{T}$ has a fixed-point in $Y$. This is equivalent to the existence part of our theorem. We observe indeed that $Y$ is a closed and convex subset of $H^{1}(\Omega)$. By the Schauder Fixed-Point Theorem it suffices to show that:

(a) $\mathcal{T}(Y) \subset Y$.

(b) The mapping $\mathcal{T}$ is compact.

Property (a) has been established in Steps 1 and 2, and Property (b) follows by parabolic regularity.

Step 4. - Once a time-periodic orbit is found in the set $Y,(3.13)$ follows using the standard form of the Csiszár-Kullback inequality (1.9).

We can now conclude this section with some remarks.

(i) A special case of Equation (3.10) is the flashing ratchet model, where $\phi_{1}$ is a sawtooth asymmetric potential (see ${ }^{20}$ for an example) which does not depend on $t$ and $\phi_{2} \equiv 0$. 
(ii) Condition (3.11) is not purely technical. It can be shown in some cases that it is not always true. We will give an example where it is satisfied in Section 5. In an interval (i.e. for $d=1$ ) of length $L$, the optimal value of $\Lambda_{0,1}^{(T)}$ (in case $\phi \equiv 0$ and $\sigma \equiv 1)$ is

$$
\Lambda_{0,1}^{(T)}=C L^{-2}
$$

for some $C>0$, so that, for an unbounded interval $\Lambda_{0,1}^{(T)}=0$.

(iii) The case $\sigma \equiv 0$ on some subinterval of $(0, T)$ can be covered by our method. Adaptations of the proof are left to the reader.

(iv) It is natural to ask if one can use directly $\mathrm{e}_{\psi_{p}}$ with $p>1$ instead of $\mathrm{e}_{\psi_{1}}$. The estimate now reads

$$
\frac{d}{d t} \mathrm{e}_{\psi_{p}}\left[u \mid u_{\phi, \sigma}\right]=-\mathrm{I}_{\psi_{p}}\left[u \mid u_{\phi, \sigma}\right]-\int_{\Omega}\left[\left(\frac{u}{u_{\phi, \sigma}}\right)^{p}-1\right]\left(u_{\phi, \sigma}\right)_{t} d x .
$$

Since the last term of the right hand side can be bounded by

$$
\begin{aligned}
\left|\int_{\Omega}\left[\left(\frac{u}{u_{\phi, \sigma}}\right)^{p}-1\right]\left(u_{\phi, \sigma}\right)_{t} d x\right|= & \left|\int_{\Omega}\left(\frac{u}{u_{\phi, \sigma}}\right)^{p}\left(u_{\phi, \sigma}\right)_{t} d x\right| \\
& \leq\left[(p-1) \mathrm{e}_{\psi_{p}}\left[u \mid u_{\phi, \sigma}\right]+M\right] K_{\phi, \sigma},
\end{aligned}
$$

it seems that the method can be extended to the case $p>1$ only under a smallness assumption, namely under the condition that $(p-1) K_{\phi, \sigma}$ is smaller than

$$
\inf _{(x, t) \in \Omega \times[0, T)} \inf _{u} \frac{\mathrm{I}_{\psi_{p}}\left[u \mid u_{\phi, \sigma}\right]}{\mathrm{e}_{\psi_{p}}\left[u \mid u_{\phi, \sigma}\right]} .
$$

\section{Diffusion equations with highly degenerate diffusion coefficients}

Consider the drift-diffusion equation

$$
u_{t}=\nabla \cdot[\sigma(|x|) \nabla u+\beta x u] \quad \forall(x, t) \in \Omega \times \mathbb{R}^{+},
$$

where $\Omega$ is a domain in $\mathbb{R}^{d}, d \geq 1, \sigma$ is a diffusion coefficient which is not positive everywhere, and $\beta$ is a positive parameter. The point we want to investigate here corresponds to the case of a degeneracy which is stronger than the one studied in Section 2, typically $\sigma(|x|)=|x|^{\alpha}$ with $\alpha>2$. We are not going to give any general result in this section, but simply illustrate a few possible behaviors by some examples.

\subsection{Example of a simple drift without diffusion: concentration}

If $\sigma \equiv 0$, the solution is explicit, as it is easily shown using the characteristics. Let

$$
\left\{\begin{array}{l}
\frac{d X}{d t}=-\beta X \\
X(0)=x
\end{array} \Longleftrightarrow X(t)=x e^{-\beta t}\right.
$$


and $J(t):=e^{-d \beta t}$. If $u$ is a solution of (4.1) with $\sigma \equiv 0$ and initial data $\bar{u}_{0} \in L_{+}^{1}(\Omega)$, then $v(x, t):=J(t) u(X(t), t)$ satisfies

$$
v_{t}=\left(u_{t}-\nabla \cdot[\beta x u]\right)_{\mid x=X(t), t}=0
$$

so that

$$
J(t) u(X(t), t)=\bar{u}_{0}(x) \quad \Longleftrightarrow \quad u(x, t)=e^{d \beta t} \bar{u}_{0}\left(e^{\beta t} x\right) .
$$

An approximation method (see below example 4.2) then shows that if the initial condition is

$$
u_{0}=m_{0} \delta+\bar{u}_{0}
$$

where $\delta$ is the Dirac delta distribution centered at $0 \in \Omega, m_{0} \geq 0$ and $\bar{u}_{0} \in L_{+}^{1}(\Omega)$, then

$$
u(x, t)=m_{0} \delta+e^{d \beta t} \bar{u}_{0}\left(e^{\beta t} x\right) \quad \forall(x, t) \in \Omega \times \mathbb{R}^{+}
$$

is also a solution in the sense of distributions.

\subsection{Example of measure valued solutions in case $\alpha>2$}

For simplicity, assume that $\sigma(|x|)=|x|^{\alpha}$ and $\Omega$ is a simply connected domain contained in a ball $B(0, R)$, with a radius $R>0$ sufficiently small, i.e., such that

$$
\gamma=\beta-\frac{1}{2}(d+\alpha) R^{\alpha-2}>0 .
$$

We are interested in understanding the consequences of the degeneracy of the diffusion coefficient at $x=0$.

Proposition 4.1. Under the above assumptions, let $u_{0}=m_{0} \delta+\bar{u}_{0}$, with $\bar{u}_{0} \in$ $L_{+}^{1}(\Omega), \bar{u}_{0}\left(|x|^{2}+\log \bar{u}_{0}\right) \in L^{1}(\Omega)$, and assume that $\alpha>2$. Then there exists a unique global weak solution of (4.1) with boundary conditions (2.2) and initial datum $u_{0}$ such that the non-singular part $u-m_{0} \delta$ belongs to $C^{0}\left(\mathbb{R}^{+}, L^{1}(\Omega)\right)$. Moreover the solution fully concentrates as $t \rightarrow+\infty$ :

$$
u(x, t) \rightarrow\left(m_{0}+\int_{\Omega} \bar{u}_{0} d x\right) \delta,
$$

in the sense of measures.

Proof. Since the equation is linear, we can treat the singular component and the $L^{1}$ component of the solution independently.

As for the singular component, consider a smooth nonnegative function $\phi$ with compact support such that $\int_{\Omega} \phi d x=1$. It is easy to see that $\phi_{\epsilon}(x)=\epsilon^{-d} \phi(x / \epsilon)$ is such that

$$
\nabla \cdot\left[\sigma(|x|) \nabla \phi_{\epsilon}+\beta x \phi_{\epsilon}\right] \rightarrow 0 \quad \text { as } \epsilon \rightarrow 0
$$


in $\mathcal{D}^{\prime}(\Omega)$, so that the Dirac delta distribution centered at 0 is a stationary solution of (4.1).

Assume that $m_{0}=0$ or equivalently consider the non singular part of the solution. The concentration as $t \rightarrow \infty$ relies on moment estimates. Straightforward computations show that

$$
-2 \beta \int_{\Omega}|x|^{2} u(x, t) d x \leq \frac{d}{d t} \int_{\Omega}|x|^{2} u(x, t) d x \leq-2 \gamma \int_{\Omega}|x|^{2} u(x, t) d x,
$$

which proves the exponential decay of $\int_{\Omega}|x|^{2} u(x, t) d x$ as $t \rightarrow \infty$. The global existence of a solution with the claimed regularity easily follows from standard results on parabolic equations.

Notice that the results of Proposition (4.1) are compatible with the observation that the zero flux solution $u_{\infty}(x)=\exp \left(-\beta\left(|x|^{2-\alpha}-1\right) /(\alpha-2)\right)$ is not integrable if $\alpha>2$.

\subsection{Example of a decentered drift: integrable asymptotic solution}

The situation is different if the drift force tends to concentrate the solution at a point where the diffusion does not degenerate, at least in dimension $d=1$. Here we assume that $\sigma(|x|)=|x|^{\alpha}$ if $|x| \leq 1$ and $\sigma(|x|)=1$ otherwise. The $L^{1}$-boundedness of the zero flux solution can indeed be restored as shown by the following example. Let $d=1$ and consider the equation

$$
u_{t}=\frac{d}{d x}\left[\sigma(|x|) \frac{d u}{d x}+\beta\left(x-x_{0}\right) u\right]
$$

for some $x_{0} \neq 0$. Take for instance $x_{0}>0$ (of course similar results also hold for $\left.x_{0}<0\right)$ and let

$$
u_{\infty}(x)= \begin{cases}0 & \text { if } \quad x<0 \\ C e^{\beta \int_{x_{0}}^{x} \frac{x_{0}-s}{\sigma(s)} d s} & \text { if } \quad x>0\end{cases}
$$

Since $\log u_{\infty} \sim-\frac{\beta}{\alpha-1}|x|^{1-\alpha}$ as $x \rightarrow 0_{+}$and $\log u_{\infty} \sim-\frac{\beta}{2}|x|^{2}$ as $x \rightarrow+\infty, u_{\infty}$ is integrable. Here we take the nonnegative constant $C$ such that $\int_{\mathbb{R}} u_{0} d x=\int_{\mathbb{R}} u_{\infty} d x$. For simplicity, let us consider only a regular case and assume that $u$ is of class $C^{1}$. More general situations can easily be handled up to certain technical regularizations.

Proposition 4.2. Assume that $u$ is a solution of (4.2) of class $C^{1}$, with an initial value $u_{0}$ with support in $\mathbb{R}^{+}$and $u_{0} \log \left(u_{0} / u_{\infty}\right) \in L^{1}\left(\mathbb{R}^{+}\right)$. Then $u$ converges to $u_{\infty}$.

Proof. First of all, an integration by parts shows that the mass is preserved and the support is contained in $\mathbb{R}^{+}$for any $t>0$. Next, no concentration of mass is possible at $x=0$. Otherwise, using the linearity of the equation and passing to the limit as $t \rightarrow+\infty$, this would mean that a Dirac delta distribution centered at 0 
is a stationary solution, which is impossible. We can therefore apply the relative entropy method.

$$
\frac{d}{d t} \int_{\mathbb{R}^{+}} u \log \left(\frac{u}{u_{\infty}}\right) d x=-\int_{\mathbb{R}^{+}} u\left|\frac{u^{\prime}}{u}-\frac{u_{\infty}^{\prime}}{u_{\infty}}\right|^{2} \sigma(|x|) d x
$$

The only possible limit is therefore $u_{\infty}$.

\subsection{Example of a time-dependent drift, without diffusion: concentration}

Consider now the equation

$$
u_{t}=\frac{d}{d x}\left[\beta\left(x-x_{0}(t)\right) u\right], \quad x \in \mathbb{R}, \quad t>0,
$$

without any diffusion: let $\sigma \equiv 0$ as in Example 4.1. Here we assume that $x_{0}(t) \not \equiv 0$ a.e. and, for simplicity, $d=1$. Let $X\left(t ; x, t_{0}\right)$ be the solution of the ODE

$$
\frac{d X}{d t}=-\beta\left(X-x_{0}(t)\right)
$$

with initial value $x$ at $t=t_{0}$. The solution of (4.4) is given by Duhamel's formula:

$$
X\left(t ; x, t_{0}\right)=x e^{-\beta\left(t-t_{0}\right)}+\beta \int_{t_{0}}^{t} e^{-\beta(t-s)} x_{0}(s) d s,
$$

and $u$ converges to the unique time-periodic solution of (4.4) with period $T$ if $x_{0}$ is itself time-periodic of period $T$. Such a solution is shown to exists by an elementary fixed point argument.

The solution $u(x, t)$ of $(4.3)$ is explicit and given by

$$
u(x, t)=J(t) u_{0}(X(0 ; x, t)),
$$

where $J(t)$ is the Jacobian of the transformation $x \mapsto X(0 ; x, t)$. This solution of course generalizes the one of Example 4.1, wich corresponds to $x_{0} \equiv 0$.

\subsection{Time-dependent drift and diffusion terms: a conjecture}

Although in Example 4.4 it may happen that $X(0 ; x, t)=x_{0}(t)$ for certain values of $t$, this is not the case for almost every $t$ if $x_{0}$ is time-periodic, non constant. Thus, if we add a diffusion like in Example 4.3, namely if we consider the equation

$$
u_{t}=\frac{d}{d x}\left(\sigma\left(\left|x-x_{0}(t)\right|\right) \frac{d u}{d x}+\beta\left(x-x_{0}(t)\right) u\right) \quad x \in \mathbb{R}, t>0,
$$

for some time-periodic, non constant function $x_{0}(t)$ of period $T$, we can make the

Conjecture. With the above assumptions, there is an asymptotic periodic state $u_{\infty}$ of period $T$, which is bounded in $L^{\infty}\left((0, T), L^{1}(\mathbb{R})\right)$. Moreover, $\int_{\mathbb{R}} u_{\infty}(x, t) d x=M$ does not depend on $t, u_{\infty}$ is unique up to a multiplication by a constant, and any solution $u$ with initial datum $u_{0}$ such that $\int_{\mathbb{R}} u_{0}(x) d x=M$ converges to $u_{\infty}$. 
In other words, this means that even for $\alpha>2$, if $x_{0}$ is non constant, then the solution converges to a time-dependent asymptotic state which is bounded in $L^{1}(\mathbb{R})$ for almost any $t>0$. Thus the situation is expected to be rather different from the one of Example 4.2, where concentration always occurs.

\section{The entropy - entropy production method}

In this last section, we present some considerations on the entropy - entropy production method, which is a very efficient method to prove inequalities relating the functionals $\mathbf{e}_{\psi}$ and $\mathbf{I}_{\psi}$. To start with, we show how to use it in order to prove an inequality such as (3.11) in a bounded domain. For simplicity and since the method is not original, we shall consider the simple case of a bounded interval $(d=1)$. We refer to ${ }^{5}$ for more details and to ${ }^{8,4,2}$ for an alternative method and improvements. The goal of this section is to compare the entropy - entropy production method with the method of Section 2.

Consider on $\mathbb{R}$ the potential

$$
V_{\alpha}(x)=\left\{\begin{array}{lll}
\frac{\alpha}{2}|x|^{2} & \text { if } & x<0 \\
\frac{1}{2}|x|^{2} & \text { if } & 0<x<1 \\
\frac{\alpha}{2}|x-1|^{2}+\frac{1}{2} & \text { if } & x>1
\end{array}\right.
$$

and let $\psi$ be a nonnegative $C^{4}$ convex function on $(0,+\infty)$ satisfying the admissibility conditions

$$
\begin{gathered}
\psi(1)=\psi^{\prime}(1)=0 \\
2\left(\psi^{\prime \prime \prime}\right)^{2} \leq \psi^{\prime \prime} \psi^{i v}
\end{gathered}
$$

where $\psi^{\prime \prime}, \psi^{\prime \prime \prime}$ and $\psi^{(i v)}$ respectively denote the second, third and fourth derivatives of $\psi$. Define $d \mu_{\alpha}(x):=e^{-V_{\alpha}(x)} d x$. According to ${ }^{5}$, we have the following result.

Proposition 5.1. Under the above assumptions, for any $\alpha>0$, for any $v \in$ $L_{+}^{1}\left(\mathbb{R}, d \mu_{\alpha}\right)$,

$$
\int_{\mathbb{R}} \psi(v) d \mu_{\alpha} \leq 2 \min (1, \alpha) \int_{\mathbb{R}} \psi^{\prime \prime}(v)\left|\frac{d u}{d x}\right|^{2} d \mu_{\alpha}
$$

Of course, both sides of this inequality, which is called "the convex Sobolev inequality" in ${ }^{5}$, can be infinite. For completeness, let us give a sketch of its proof. Consider the 1-dimensional Fokker-Planck type equation

$$
u_{t}=\frac{d^{2} u}{d x^{2}}+\frac{d}{d x}\left(u \frac{d}{d x} V_{\alpha}\right)
$$

with initial data $u_{0}:=v e^{-V_{\alpha}(x)}$. Let $u_{\infty}=\kappa e^{-V_{\alpha}(x)}$, where $\kappa>0$ is such that $\int_{\mathbb{R}} u d x=\int_{\mathbb{R}} u_{\infty} d x$. If the relative entropy of the initial data

$$
\mathrm{e}_{\psi}\left[u_{0} \mid u_{\infty}\right]:=\int_{\mathbb{R}} \psi\left(\frac{u_{0}}{u_{\infty}}\right) u_{\infty} d x
$$


is finite, then

$$
\frac{d}{d t} \mathrm{e}_{\psi}\left[u(\cdot, t) \mid u_{\infty}\right]=-\mathrm{I}_{\psi}\left[u(\cdot, t) \mid u_{\infty}\right]
$$

with

$$
\mathrm{I}_{\psi}\left[u \mid u_{\infty}\right]:=\int_{\mathbb{R}} \psi^{\prime \prime}\left(\frac{u}{u_{\infty}}\right)\left|\frac{d}{d x}\left(\frac{u}{u_{\infty}}\right)\right|^{2} u_{\infty} d x .
$$

This proves that $u(\cdot, t)$ converges to $u_{\infty}$ in the sense of the relative entropy:

$$
\lim _{t \rightarrow+\infty} \mathrm{e}_{\psi}\left[u(\cdot, t) \mid u_{\infty}\right]=0 .
$$

At this point we dont need to make this convergence very precise: it holds almost everywhere with respect to $u_{\infty} d x$ and in $L^{p}$ for some $p \in[1,2]$ in the cases of interest for this paper. The only crucial point is to get that

$$
\mathrm{I}_{\psi}\left[u \mid u_{\infty}\right]=0 \quad \Longrightarrow \quad \mathrm{e}_{\psi}\left[u \mid u_{\infty}\right]=0
$$

Next, by computing

$$
\begin{aligned}
& \frac{d}{d t}\left(\mathrm{I}_{\psi}\left[u(\cdot, t) \mid u_{\infty}\right]-\lambda \mathrm{e}_{\psi}\left[u(\cdot, t) \mid u_{\infty}\right]\right) \\
& =\int_{\mathbb{R}} \psi^{\prime \prime}\left(\frac{u}{u_{\infty}}\right)\left|\frac{d}{d x}\left(\frac{u}{u_{\infty}}\right)\right|^{2}\left(\lambda-2 V_{\alpha}^{\prime \prime}\right) u_{\infty} d x-\int_{\mathbb{R}} \operatorname{Tr}(X Y) u_{\infty} d x
\end{aligned}
$$

where both $X$ and $Y$ are nonnegative definite matrices:

$$
X=\left(\begin{array}{cc}
2 \psi^{\prime \prime}(v) & 2 \psi^{\prime \prime \prime}(v) \\
2 \psi^{\prime \prime \prime}(v) & \psi^{(i v)}(v)
\end{array}\right) \quad \text { and } \quad Y=\left(\begin{array}{cc}
\left(v^{\prime \prime}\right)^{2} & \left(v^{\prime}\right)^{2} v^{\prime \prime} \\
\left(v^{\prime}\right)^{2} v^{\prime \prime} & \left(v^{\prime}\right)^{4}
\end{array}\right)
$$

with $v=u / u_{\infty}$, we obtain that for $\lambda=2 \inf _{\mathbb{R}} V_{\alpha}^{\prime \prime}=2 \min (1, \alpha)$, for any $t \geq 0$,

$$
\frac{d}{d t} \mathrm{I}_{\psi}\left[u(\cdot, t) \mid u_{\infty}\right] \leq-\lambda \mathrm{I}_{\psi}\left[u(\cdot, t) \mid u_{\infty}\right]
$$

After an integration from $t$ to $\infty$, this proves that

$$
\begin{aligned}
\mathrm{I}_{\psi}\left[u_{0} \mid u_{\infty}\right]-\lambda \mathrm{e}_{\psi}\left[u_{0} \mid u_{\infty}\right] & \geq \mathrm{I}_{\psi}\left[u(\cdot, t) \mid u_{\infty}\right]-\lambda \mathrm{e}_{\psi}\left[u(\cdot, t) \mid u_{\infty}\right] \\
& \geq \lim _{t \rightarrow+\infty}\left(\mathrm{I}_{\psi}\left[u(\cdot, t) \mid u_{\infty}\right]-\lambda \mathrm{e}_{\psi}\left[u(\cdot, t) \mid u_{\infty}\right]\right)=0,
\end{aligned}
$$

which is equivalent to (5.2). To justify these computations, we can regularize $V_{\alpha}$, use smooth functions which are dominated by $C u_{\infty}$ for a sufficiently large $C>0$ and conclude by density.

By taking the limit $\alpha \rightarrow+\infty$ and using Lebesgue's theorem of dominated convergence in (5.1), we obtain

$$
\int_{0}^{1} \psi(v) u_{\infty} d x \leq 2 \int_{0}^{1} \psi^{\prime \prime}(v)\left|\frac{d v}{d x}\right|^{2} u_{\infty} d x
$$

where $u_{\infty}(x)=e^{-x^{2} / 2}$. The functions $\psi_{p}(v)=\left(v^{p}-1-p(v-1)\right) /(p-1)$ for any $p \in(1,2]$ and $\psi_{1}(v)=v \log v-(v-1)$ are convex and admissible in the sense of 
(5.1). Using a Holley-Stroock perturbation lemma as in the proof of Theorem 2.1, this proves that, for any function $v$ bounded away from 0 ,

$$
\mathrm{e}_{\psi}[u \mid v] \leq \mathcal{C} \mathrm{I}_{\psi}[u \mid v]
$$

for some strictly positive constant $\mathcal{C}$. This result can be extend without difficulties to any convex domain in $\mathbb{R}^{d}, d \geq 1$.

One may wonder if the entropy - entropy production method applies when the diffusion coefficient degenerates. Dealing directly with bounded domains is not easy, because the method involves several integrations by parts and one has to control boundary terms. Let us give an example in $\mathbb{R}^{d}$. Consider as in Section 2 the case

$$
\sigma(x)=|x|^{\alpha}, \quad A(x):=-\log u_{\infty}(x)=\frac{|x|^{2-\alpha}}{2-\alpha}
$$

for some $\alpha \in(0,2)$. According to ${ }^{5}$, the basic assumption of the entropy - entropy production method is the so-called generalized Bakry-Emery condition: for some $\lambda>0$,

$$
\begin{aligned}
& {\left[\frac{d-3}{2} \frac{\sigma^{\prime}}{r}-\frac{1}{2} \sigma^{\prime} A^{\prime}+\frac{1}{2} \sigma^{\prime \prime}+\sigma \frac{A^{\prime}}{r}\right] \mathbb{I}_{d}} \\
& \quad+\left[\sigma^{\prime} A^{\prime}-\frac{d-2}{4} \frac{\left(\sigma^{\prime}\right)^{2}}{\sigma}+\frac{\sigma^{\prime}}{r}-\sigma^{\prime \prime}+\sigma\left(A^{\prime \prime}-\frac{A^{\prime}}{r}\right)\right] \mathbb{S}(x) \geq \lambda \mathbb{I}_{d}
\end{aligned}
$$

for any $x \in \mathbb{R}^{d}$, in the sense of positive matrices. Here $\mathbb{I}_{d}$ is the identity matrix in $\mathbb{R}^{d} \times \mathbb{R}^{d}, \mathbb{S}(x)=\left(x^{i} x^{j} / r^{2}\right)_{i, j=1,2, \ldots d}, r=|x|$ and we write abusively $\sigma(x)=\sigma(r)$, $\nabla \sigma(x)=\sigma^{\prime}(r) x / r$, etc. The generalized Bakry-Emery condition means

$$
\left[\alpha(d-4+\alpha) r^{\alpha-2}+2-\alpha\right] \mathbb{I}_{d}+\frac{\alpha}{2}[8-(d+2) \alpha] r^{\alpha-2} \mathbb{S}(x) \geq 2 \lambda \mathbb{I}_{d}
$$

for any $x \in \mathbb{R}^{d}$. The eigenvalues of the left hand side are

$$
\alpha(d-4+\alpha) r^{\alpha-2}+2-\alpha \quad \text { and } \quad(2-\alpha)\left[1+\frac{d}{2} \alpha r^{2-\alpha}\right]
$$

which are both positive for all $r>0$, under the condition

$$
\max \{0,4-d\}<\alpha<2 .
$$

Hence the entropy - entropy production method can be applied for $d \geq 3$, but with some restrictions for $d=3$ and not at all if $d \leq 2$. When it applies, the result however holds even if $p=1$, with the notations of Section 2 .

More general diffusion coefficients can also be considered under the conditions

$$
(d-3) \frac{\sigma^{\prime}}{r}-\sigma^{\prime} A^{\prime}+\sigma^{\prime \prime}+2 \sigma \frac{A^{\prime}}{r}>\lambda
$$

and

$$
(d-1) \frac{\sigma^{\prime}}{r}+\sigma^{\prime} A^{\prime}-\sigma^{\prime \prime}-\frac{1}{2}(d-2) \frac{\left(\sigma^{\prime}\right)^{2}}{\sigma}+2 \sigma A^{\prime \prime}>\lambda \quad \forall r \in \mathbb{R}^{+}
$$


The entropy - entropy production method shows that

$$
\Sigma_{p}[u] \leq \frac{1}{2 \lambda} \mathrm{I}_{p}[u] .
$$

By Holley-Stroock type perturbation arguments, it is possible to perturb $A$ and $\sigma, c f .{ }^{24}$ for the logarithmic entropy $\sigma_{1}$ and $\S 3.3$ of ${ }^{5}$ for general admissible entropies. Also see ${ }^{4}$ for some recent refined results in this direction. As a special case, the above inequality also holds for convex domains $\Omega \subset \mathbb{R}^{d}$. In all these cases, the estimate is obtained uniformly with respect to $p \in[1,2]$. An alternative perturbative approach has recently been developed by Cattiaux in ${ }^{14}$ and Carlen and Loss in ${ }^{11}$, in order to derive logarithmic Sobolev type inequalities corresponding to $p=1$ from the spectral gap inequality corresponding to $p=2$. Such results have been extended in ${ }^{7}$ to all convex entropies $\Sigma_{p}$ with $p \in[1,2)$.

Acknowledgments. J.D. would like to acknowledge the hospitality of the Department of Mathematics and Statistics at the University of Victoria, where some of the work was done, and support by grant \# 7847 of the Natural Sciences and Engineering Research Council of Canada. M.K. would like to thank for the hospitality he received during his visit at the Université Paris-Dauphine when a part of this work was completed. He was also partly supported by FONDECYT grant \# 105031. This research has also been supported by the EU financed network HPRN-CT-2002-00282.

(C) 2005 by the authors. This paper may be reproduced, in its entirety, for non-commercial purposes.

\section{References}

1. A. Ajdari And J. Prost, Mouvement induit par un potentiel périodique de basse symétrie: dielectrophorese pulse, C. R. Acad. Sci. Paris, Série II, 315 (1992), p. 1653.

2. A. Arnold, J.-P. Bartier, And J. Dolbeault, Interpolation between logarithmic Sobolev and Poincaré inequalities, tech. rep., Ceremade, preprint no. 0528, 2005.

3. A. Arnold, J. A. Carrillo, L. Desvillettes, J. Dolbeault, A. Jüngel, C. LeDerman, P. A. Markowich, G. Toscani, and C. Villani, Entropies and equilibria of many-particle systems: an essay on recent research, Monatsh. Math., 142 (2004), pp. 35-43.

4. A. Arnold And J. Dolbeault, Refined convex Sobolev inequalities, J. Funct. Anal., 225 (2005), pp. 337-351.

5. A. Arnold, P. Markowich, G. Toscani, and A. Unterreiter, On convex Sobolev inequalities and the rate of convergence to equilibrium for Fokker-Planck type equations, Comm. Partial Differential Equations, 26 (2001), pp. 43-100.

6. R. Astumian, Thermodynamics and kinetics of a brownian motor, Science, 276 (1997), pp. 917-922.

7. J.-P. Bartier And J. Dolbeault, Convex Sobolev inequalities and spectral gap, tech. rep., Ceremade, preprint no. 0513, 2005.

8. W. Beckner, A generalized Poincaré inequality for Gaussian measures, Proc. Amer. Math. Soc., 105 (1989), pp. 397-400.

9. S. G. Bobkov, I. Gentil, And M. Ledoux, Hypercontractivity of Hamilton-Jacobi equations, J. Math. Pures Appl. (9), 80 (2001), pp. 669-696. 
10. M. J. CÁceres, J. A. Carrillo, And J. Dolbeault, Nonlinear stability in $L^{p}$ for a confined system of charged particles, SIAM J. Math. Anal., 34 (2002), pp. 478-494.

11. E. CARLEN AND M. Loss, Logarithmic Sobolev inequalities and spectral gaps, in Recent advances in the theory and applications of mass transport, vol. 353 of Contemp. Math., Amer. Math. Soc., Providence, RI, 2004, pp. 53-60.

12. J. A. Carrillo, A. Jüngel, P. A. Markowich, G. Toscani, and A. UnterreITER, Entropy dissipation methods for degenerate parabolic problems and generalized Sobolev inequalities, Monatsh. Math., 133 (2001), pp. 1-82.

13. F. CATRINA AND Z.-Q. WANG, On the Caffarelli-Kohn-Nirenberg inequalities: sharp constants, existence (and nonexistence), and symmetry of extremal functions, Comm. Pure Appl. Math., 54 (2001), pp. 229-258.

14. P. Cattiaux, Hypercontractivity for perturbed diffusion semi-groups, Preprint, (2004).

15. F. Chalub And M. Souza, The continuous Moran process. In preparation, 2005.

16. M. Сhipot, D. Kinderlehrer, And M. KowalczyK, A variational principle for molecular motors, Meccanica, 38 (2003), pp. 505-518. Dedicated to Piero Villaggio on the occasion of his 70th birthday.

17. I. CSISZÁR, Information-type measures of difference of probability distributions and indirect observations, Studia Sci. Math. Hungar., 2 (1967), pp. 299-318.

18. M. Del Pino AND J. Dolbeault, Best constants for Gagliardo-Nirenberg inequalities and applications to nonlinear diffusions, J. Math. Pures Appl. (9), 81 (2002), pp. $847-875$.

19. J. Dolbeault And R. IllneR, Entropy methods for kinetic models of traffic flow, Commun. Math. Sci., 1 (2003), pp. 409-421.

20. J. Dolbeault, D. Kinderlehrer, And M. Kowalczyk, Remarks about the flashing rachet, in Partial differential equations and inverse problems, vol. 362 of Contemp. Math., Amer. Math. Soc., Providence, RI, 2004, pp. 167-175.

21. W. E AND P. PALFFy-Muhoray, Orientational ratchets and angular momentum balance in the Janossy effect, Mol. Cryst. Liq. Cryst., 320 (1998), pp. 193-206.

22. L. Gross, Logarithmic Sobolev inequalities, Amer. J. Math., 97 (1975), pp. 1061-1083.

23. M. Günther, A. Klar, T. Materne, And R. Wegener, Multivalued fundamental diagrams and stop and go waves for continuum traffic flow equations, SIAM J. Appl. Math., 64 (2003/04), pp. 468-483.

24. R. Holley AND D. Stroock, Logarithmic Sobolev inequalities and stochastic Ising models, J. Statist. Phys., 46 (1987), pp. 1159-1194.

25. R. Illner, A. Klar, AND T. MATERne, On Vlasov-Fokker-Planck type kinetic models for multilane traffic flow, in Proceedings of the 23rd International Symposium on Rarefied Gas Dynamics, P. Muntz and A. Ketsdever, eds., 2003.

26. — Vlasov-Fokker-Planck models for multilane traffic flow, Commun. Math. Sci., 1 (2003), pp. 1-12.

27. R. Jordan, D. Kinderlehrer, And F. Otto, The variational formulation of the Fokker-Planck equation, SIAM J. Math. Anal., 29 (1998), pp. 1-17.

28. D. Kinderlehrer AND M. KOWALCZYK, Remarks about diffusion mediated transport, Ricerche Mat., 49 (2000), pp. 305-318. Contributions in honor of the memory of Ennio De Giorgi (Italian).

29. — Diffusion-mediated transport and the flashing ratchet, Arch. Ration. Mech. Anal., 161 (2002), pp. 149-179.

30. S. KULlBACK, On the convergence of discrimination information, IEEE Trans. Information Theory, IT-14 (1968), pp. 765-766.

31. Y. OKADA AND N. HIROKAWA, A processive single-headed motor: kinesin superfamily protein KIF1A, Science, 283 (1999). 
32. - Mechanism of the single headed processivity: diffusional anchoring between the $K$-loop of kinesin and the $C$ terminus of tubulin, Proc. Nat. Acad. Sciences, 7 (2000), pp. 640-645.

33. F. Отто, The geometry of dissipative evolution equations: the porous medium equation, Comm. Partial Differential Equations, 26 (2001), pp. 101-174.

34. V. Rădulescu, D. Smets, And M. Willem, Hardy-Sobolev inequalities with remainder terms, Topol. Methods Nonlinear Anal., 20 (2002), pp. 145-149.

35. G. Toscani, Sur l'inégalité logarithmique de Sobolev, C. R. Acad. Sci. Paris Sér. I Math., 324 (1997), pp. 689-694.

36. A. Unterreiter, A. Arnold, P. Markowich, and G. Toscani, On generalized Csiszár-Kullback inequalities, Monatsh. Math., 131 (2000), pp. 235-253.

37. C. Villani, Topics in optimal transportation, vol. 58 of Graduate Studies in Mathematics, American Mathematical Society, Providence, RI, 2003. 